\title{
Solitary waves and modulation instability with the influence of fractional derivative order in nonlinear left-handed transmission line
}

\section{Djidere Ahmadou}

Universite de Ngaoundere

\section{Alphonse Houwe}

Universite de Maroua

Justin Mibaile

Universite de Maroua

\section{Gambo Betchewe}

Universite de Maroua

\section{Serge Y. Doka}

Universite de Ngaoundere

\section{Kofane T. Crepin}

University of Yaounde I: Universite de Yaounde I

Mustafa Inc ( $\nabla$ minc@firat.edu.tr)

Firat Universitesi https://orcid.org/0000-0003-4996-8373

\section{Research Article}

Keywords: Solitary waves, modulation instability, fractional derivative order, nonlinear left-handed transmission line

Posted Date: March 29th, 2021

DOI: https://doi.org/10.21203/rs.3.rs-261753/v1

License: (c) (1) This work is licensed under a Creative Commons Attribution 4.0 International License. Read Full License

Version of Record: A version of this preprint was published at Optical and Quantum Electronics on July 14th, 2021. See the published version at https://doi.org/10.1007/s11082-021-03055-y. 


\title{
Solitary waves and modulation instability with the influence of fractional derivative order in nonlinear left-handed transmission line
}

\author{
Djidere Ahmadou ${ }^{\mathrm{a}, *}$, Houwe Alphonse $^{\mathrm{b}}$, Mibaile Justin $^{\mathrm{c}}$, Gambo Betchewe $^{\mathrm{b}}$, \\ Doka Yamigno Serge ${ }^{\mathrm{a}}$, Kofane Timoleon Crepin ${ }^{\mathrm{d}}$, Mustafa Inc ${ }^{\mathrm{e}, \mathrm{f}}$ \\ ${ }^{a}$ Department of Physics, Faculty of Science, The University of Ngaoundere, P.O. Box 454, \\ Ngaoundere, Cameroon \\ ${ }^{b}$ Department of Physics, Faculty of Science, The University of Maroua, P.O. Box 814, \\ Cameroon \\ ${ }^{c}$ Higher Teachers' Training College of Maroua, The University of Maroua, P.O. Box. 46, \\ Maroua, Cameroon \\ ${ }^{d}$ Department of Physics, Faculty of Science, The University of Yaounde I, P.O. Box 812, \\ Yaounde, Cameroon \\ ${ }^{e}$ Department of Mathematics, Firat University, 23119 Elazig, Turkey \\ ${ }^{f}$ Department of Medical Research, China Medical University Hospital, China Medical \\ University, Taichung, Taiwan
}

\begin{abstract}
The resolution of the reduced fractional nonlinear Schrödinger equation obtained from the model describing the wave propagation in the left-handed nonlinear transmission line presented by Djidere et al recently, allowed us in this work through the Adomian decomposition method (ADM) to highlight the behavior and to study the propagation process of the dark and bright soliton solutions with the effect of the fractional derivative order as well as the Modulation Instability gain spectrum (MI) in the LHNLTL. By inserting fractional derivatives in the sense of Caputo, we used ADM to structure the approximate solitons solutions of the fractional nonlinear Schrödinger equation reduced with fractional derivatives. The pipe is obtained from the bright and dark soliton by the fractional derivatives order (see Figures 2 and 5). By the bias of MI gain spectrum the instability zones occur when the value of the fractional derivative order tends to 1 . Furthermore, when the fractional derivative order takes small
\end{abstract}

\footnotetext{
*Corresponding author.

Email address: djidereahmadou@gmail.com, minc@firat.edu.tr (Djidere Ahmadou)
} 
values, stability zones appear. These results could bring new perspectives in the study of solitary waves in left-handed metamaterials, as the memory effect could have a better future for the propagation of modulated waves because we also show in this article that the stabilization of zones of the dark and bright solitons could be described by a fractional nonlinear Schrödinger equation with small values of fractional derivatives order. In addition, the obtained significant results are new and could find applications in many research areas such as in the field of information and communication technologies.

Keywords: Solitary waves, modulation instability, fractional derivative order, nonlinear left-handed transmission line

\section{Introduction}

The evolution of digital electronics and communication has made enormous advances, particularly in the transmission of information. In previous years, coaxial cables were mainly used, which transported signals with a lot of losses.

5 To overcome this difficulty, researchers have introduced a recent class of materials, called "metamaterials", to simplify the size of components and thus reduce maximum losses by making them more efficient. As a result, the micro line ribbons [1] were born. The expression "metamaterial" was first mentioned in the field of optics by the Russian physicist [2] when he theoretically introduced the concept of left-handed materials and its realization by $[3]$.

In this current work, we focus on the study of the propagation of dark and bright solitons in the left-handed nonlinear transmission line (LHNLTL) by the Adomian decomposition method with the effect of the fractional derivative order $(\alpha)$. Recently in literature fractional differential equations have been of great interest. Nowadays, there has been considerable attention in non linear evolution equations having fractional differential equations. At the very beginning, there were hardly any practical applications of fractional calculus, and it was highly regarded as an obscure field with only rarely or not at all advantageous mathematical manipulations. For almost 30 years, the paradigm began to move 
from simple mathematical formulations to applications in several fields. For previous years, fractional calculus was used and applied to almost all areas of science, engineering and mathematics. Fields of application of fractional differentiation and fractional integration are already well established, others have just begun and will continue. Fractional computation applications are found

25 in turbulence and fluid dynamics, stochastic dynamical system, plasma physics and controlled thermonuclear fusion, nonlinear control theory, image processing, nonlinear biological systems, astrophysics [4, 5], [6, 7], 8, 9], 10, 11, 12, , 13, 14]. Nevertheless, some historical summaries of the developments of the fractional calculation developments used can be found in the works of [4, 5] and 6]. Several methods to obtain exact solutions or even soliton solutions to nonlinear partial differential equations have been proposed, such as the Bäcklund transformation method [15, the Hirota's bilinear method [16], the inverse scattering transform method [17, the extended tanh method [18, the Adomian approximation [19], the variational method [20], the variational iteration method 21], the various 35 Lindstedt-Poincare methods [22], the Adomian decomposition method (ADM) [23, 24, 25, 26, 27], the F-expansion method [28], the exp-function method [29], the homotopy perturbation method [30, 31] and the results on the acquired solitary waves were successful thanks to the different mathematical methods recently used such as the Darboux transformation [32, 33, 34, 35],Hirota-Riemann 40 method [36],numerical simulation [37, Hirota method 38].

The main purpose of this work is to investigate the behavior of the dark and bright solitons by a nonlinear evolution equation with fractional order describing the waves propagation in nonlinear left-handed electrical transmission line giving by 39,40 ,

$$
\frac{\partial \mathrm{U}}{\partial z}-\beta_{2} \frac{j^{-3 \alpha}}{(2 \alpha) !} \frac{\partial^{2 \alpha} \mathrm{U}}{\partial t^{2 \alpha}}-\left(j+j^{-\alpha}\right) \beta_{0} U+T_{4}|U|^{2} U=0
$$

Thus, in this article we apply the Adomian decomposition method (ADM) to this equation (1) of [39, 40] to study the solutions of dark and bright solitons with the effect of fractional derivatives order and initial conditions. The details 
of the set of nonlinear evolution equations are specified [39, 40]. Here $(\alpha)$ is the fractional derivative order and $0<\alpha \leq 1$. The first step will give the overview of the fractional derivative order. The next section will give the preliminaries and the description of the Adomian decomposition method (ADM). Later the behavior of the bright and dark solitons with the effect of derivative order will be examined. Some graphical representation will follow to highlight physical phenomena of the nonlinear left-handed transmission line. The last section will conclude the work.

\subsection{Overview of the fractional derivative order}

In recent years, several properties aimed at facilitating the use of the fractional derivative have been the subject of various scientific publications, followed by concordant results. It should be remembered that some of the usual properties have not known exclusive salvation. Among the current properties, here are few that will promote the use of the system to be learned with the appropriate derivatives of the order $(\alpha)$ [1, 26, 27] below

$$
\frac{d^{\alpha} k(t)}{d t^{\alpha}}=\lim _{\epsilon \rightarrow+\infty} \frac{k\left(t+\epsilon t^{1-\alpha}\right)-k(t)}{\epsilon}, \quad k:(0, \infty) \rightarrow R
$$

With $t>0, \alpha \in(0,1]$.

Then if $k$ is $\alpha$-differentiable in some $(0, c), c>0$ and $\lim _{t \rightarrow 0^{+}} D^{\alpha}(k)(t)$ exists, 65 however by accuracy

- $D^{\alpha}(g)(0)=\lim _{t \rightarrow 0^{+}} D^{\alpha}(k)(t)$.

Taking into account $42,43,26,27$, the following relevant properties of fractional derivatives are presented as follows:

- $D_{t}^{\alpha}(c g+e k)=c D_{t}^{\alpha} g+e D_{t}^{\alpha} k$, and $c, e \epsilon R$.

- $D_{t}^{\alpha}\left(t^{\mu}\right)=\mu t^{\mu-\alpha} \quad$ and $\quad \mu \epsilon R$.

- $D_{t}^{\alpha}(g k)=k D_{t}^{\alpha}(g)+f D_{t}^{\alpha}(k)$. 
- $D_{t}^{\alpha}\left(\frac{g}{k}\right)=. \frac{k D_{t}^{\alpha}(g)-f D_{t}^{\alpha}(k)}{g^{2}}$.

- So if $g$ is differentiable, $D_{t}^{\alpha}(g)(t)=t^{1-\alpha} \frac{d g}{d t}$.

- if $g, k:(0, \infty) \rightarrow R$ is differentiable and $\alpha$ the differentiable functions: $D_{t}^{\alpha}(g o k)(t)=t^{1-\alpha} k^{\prime}(t) g^{\prime}(k(t))$.

\section{Preliminaries and Adomian decomposition method (ADM)}

\subsection{Preliminaries}

Considering equation (1), we can obtain the following nonlinear Schrödinger equation with nonlocal operator of Riemann-Liouville as follows

$$
\beta_{2} \frac{j^{-3 \alpha}}{(2 \alpha) !} \mathrm{ID}_{t}^{2 \alpha} U-\frac{\partial \mathrm{U}}{\partial z}+\left(j+j^{-\alpha}\right) \beta_{0} U-T_{4}|U|^{2} U=0,
$$

80

with respectively $\beta_{0}=1 /\left(\omega_{0}^{\alpha} \sqrt{L_{L} C_{0}}\right)$ the phase velocity, $\beta_{2}=2 /\left(\omega_{0}^{3 \alpha} \sqrt{L_{L} C_{L}}\right)$ the second order dispersion constant and $T_{4}=\rho^{2} / 4\left(\omega_{0} \sqrt{L_{L} C_{0}}\right)^{3}$ the Kerr nonlinear part of the left-handed nonlinear transmission line (LHNLTL), where $(\rho)$ is a constant of the polarization, and $0<\alpha \leq 1$ [39], while the nonlocal operator $\mathrm{ID}_{t}^{2 \alpha}$ in the sence of Riemann-Liouville read as

$$
\mathrm{ID}_{t}^{\sigma} U(z, t)=\frac{1}{\Gamma(2-\sigma)} \frac{d^{2}}{d t^{2}} \int_{0}^{t}(t-s)^{1-\sigma}\left[U(z, s)-U(z, 0)-s \frac{d}{d s} U(z, 0)\right] d s .
$$

${ }_{85}$ For simplification purpose, we set: $A=\beta_{2} \frac{j^{-3 \alpha}}{(2 \alpha) !}, B=\left(j+j^{-\alpha}\right) \beta_{0}, \sigma=2 \alpha$, so that equation (3) becomes

$$
A \mathrm{ID}_{t}^{\sigma} U-\partial_{z} U+B U-T_{4}|U|^{2} U=0, \quad U(z, 0)=f(z), \quad j=\sqrt{-1} .
$$

This part can be found in several chapters of physics, especially plasma physics, nonlinear optics, superconductivity, etc. However, cubic nonlinearity is the most common nonlinearity in applications. Nevertheless, this is a simplified model for examining Bose-Einstein condensates, Kerr medium and nonlinear optical anomalous waves in the ocean [44, 45, 46, 47, 48, 49. 
Thus, 44] studied a nonlinear Schrödinger equation such as the equation (1) of [39] by applying the differential transformation method to obtain its approximate solution.

Let's consider equation (1) in this work and we grant several definitions and basic properties of the theory of fractional calculus that are further applied in these papers [5, 6. Using fractional calculus in the sense of Riemann-Liouville and then the Adomian decomposition method with the effect of the fractional derivative order, and we get this fractional nonlinear Schrödinger equation of the form equation (3) then equation (4).

For the purpose of simplification, equation (3) can be reduced to equation (5). It is worthwhile mentioning that equation (5) is a complex differential equation, with complex modulus term $|U|^{2}$, which is nonlinear. Therefore an appropiate method to sort-out solution of equation (5) is needed. We shall use in the following the Adomian decomposition method.

\subsection{Adomian decomposition method (ADM)}

The Adomian decomposition method is an iteration method for solving linear, nonlinear, algebraic equations. The main advantage of this ADM is to provide solutions of an infinite series, which converges quickly to the solution exact. The ADM consists in dividing a given equation into linear and nonlinear parts, inverting the higher-order derivative operator.

\subsubsection{Description of the Adomian decomposition method}

Consider a general nonlinear equation in canonical form as

$$
£ U+V U+W U=0,
$$

where the linear part of a general nonlinear equation (6) is $£(U)+V(U)$. $£(U)$ is a linear operator easily invertible, $V(U)$ is the part equation and the nonlinear term is represented by $W(U)$.

The technique consists of decomposition the linear and nonlinear parts of equation (6) to apply linear operator of the highest-ordered derivation of terms. 
Notice here, $£$ is a linear operator given as $£=\frac{d}{d t}($.$) . Its inverse £^{-1}$ is defined ${ }_{120} \quad$ as $£^{-1}=\int_{0}^{t}() d$.$s .$

If we apply inverse operator $£^{-1}$ in all sided of equation (6) and using the initial condition $U(z, 0)=f(z)$, we get the following equivalent equation

$$
£^{-1} £(U)=-£^{-1} V(U)-£^{-1} W(U),
$$

this $£^{-1} £(U)=U(z, t)-U(z, 0)$.

Next we shall write the linear terms broken up by an infinite series components of the functions $U(z, t)$ defined as

$$
U(z, t)=\sum_{n=0}^{\infty} U_{n}(z, t)
$$

the nonlinear term $W(U)$ can be decomposed into an infinite series of polynomials

$$
W(U)=\sum_{n=0}^{\infty} A_{n},
$$

where $A_{n}$ is called Adomian Polynomial of $U_{n}(z, t)$. Substituting equation (8) and equation (9) into equation (6) we have

$$
\begin{gathered}
\sum_{n=0}^{\infty} U_{n}=U_{0}(z, 0)-£^{-1} V \sum_{n=0}^{\infty} U_{n}-£^{-1} \sum_{n=0}^{\infty} A_{n}, \\
\left\{\begin{array}{l}
U_{0}(z, 0)=f(z), \\
U_{n+1}(z, t)=-£^{-1} V U_{n}-£^{-1} A_{n} .
\end{array}\right.
\end{gathered}
$$

We compute the elements of $U_{n}(z, t), n=0,1,2, \ldots .$. , using recursive relations:

$$
\begin{gathered}
U_{1}=-£^{-1} V U_{0}-£^{-1} A_{0}, \\
U_{2}=-£^{-1} V U_{1}-£^{-1} A_{1}, \\
U_{n+1}=-£^{-1} V U_{n}-£^{-1} A_{n} .
\end{gathered}
$$


It consist to:

135

- Show the initial condition as the first term of the solution in infinite solutions of series;

- Break down the nonlinear function, in terms of special, polynomials which is called Adomian's polynomials;

- Provide successive terms of series solution by recurrence using Adomian's polynomials as

$$
A_{n}\left(U_{0}, U_{1}, U_{2}, \ldots, U_{n}\right)=\frac{1}{n !}\left\{\frac{d^{n}}{d \lambda^{n}}\left[\left(\sum_{k=0}^{n} \lambda^{k} U_{k}\right)\left(\sum_{k=0}^{n} \lambda^{k} U_{k}^{*}\right)\left(\sum_{k=0}^{n} \lambda^{k} U_{k}\right)\right]_{\lambda=0}\right\}
$$

\section{Dark and Bright solitons solutions}

In this section it is assumed the following dark and bright expression as solutions of equation (5):

$$
\left\{\begin{array}{l}
U(z, t)=\tanh (k z-\omega t) \mathrm{e}^{j(z-v t)}, \\
U(z, t)=D \operatorname{sech}(k z-\omega t) \mathrm{e}^{j(z-v t)} .
\end{array}\right.
$$
$\left.\tanh (k z) \mathrm{e}^{j z}, U_{0}=D \operatorname{sech}(k z) \mathrm{e}^{j z}\right)$.

The next section will emphasize the behavior of the dark and bright soliton solutions by employing the Adomian decomposition method.

\subsection{Application of the Adomian decomposition method}

\subsubsection{Fractional dark soliton solutions}

We now consider equation (5) which is a fractional nonlinear Schrödinger equation. Applying the fractional Riemann-Liouville integral of order $\sigma$ to equation (5) we get: 


$$
I_{t}^{\sigma}\left[A \mathrm{ID}_{t}^{\sigma} U-\partial_{z} U+B U-T_{4}|U|^{2} U\right]=0
$$

i.e,

$$
I_{t}^{\sigma}\left(\operatorname{ID}_{t}^{\sigma} U\right)=\frac{1}{A} I_{t}^{\sigma}\left[\partial_{z} U-B U+T_{4}|U|^{2} U\right] .
$$

Now, taking into account the properties of the inverse of the fractional derivative in the sense of Riemann-Liouville, we obtain

$$
\left(I_{t}^{\sigma} \quad \mathrm{ID}_{t}^{\sigma} U\right)(t)=U(z, t)-U(z, 0)-t \frac{\partial U(z, 0)}{\partial t},
$$

setting $U(z, 0)=\tanh (k z) \mathrm{e}^{j z}=U_{0}(z)$ and $\frac{\partial U(z, 0)}{\partial t}=0$, we get:

$$
U(z, t)=U(z, 0)+\frac{1}{A} I_{t}^{\sigma}\left[\partial_{z} U-B U+T_{4}|U|^{2} U\right] .
$$

Using the $\mathrm{ADM}$ on equation 20 , we divide the given equation into linear and nonlinear parts, the higher-order derivative operator contained in the linear operator on both sides and show the initial conditions and the first term of the series solution, it is obtained:

$$
\left\{\begin{array}{l}
U_{0}(z, 0)=\tanh (k z) \mathrm{e}^{j z}, \\
U_{n+1}(z, t)=\frac{1}{A} I_{t}^{\sigma}\left[\partial_{z} U_{n}-B U_{n}+T_{4} A_{n}\right], n \geq 0 .
\end{array}\right.
$$

Where $A_{n}=|U|^{2} U$ and $U(z, 0)=f(z)$. As in the Adomian decomposition method $[23,24,25,50$, if we consider that a serial solution of the function $U(z, t)$ is provided by equation (8) and the nonlinear term $A_{n}$ can be dissociated into an infinite range of polynomials yielded by equation (9). However, components $U_{n}(z, t)$ will be calculated recursively, the $A_{n}$ are considered as the adomial polynomials of the $U_{n}$.

Some exclusive algorithms have been determined in [23, 24, 25, 51, 50] to compute the adomial polynomials of the nonlinear terms of equation 15 .

So, we have

$$
U_{n+1}(z, t)=\frac{1}{A} I_{t}^{\sigma}\left[\partial_{z} U_{n}-B U_{n}+T_{4} A_{n}\right], n \geq 0
$$


Equation 15 leads to :

$$
\begin{aligned}
& A_{0}\left(U_{0}\right)=U_{0}\left|U_{0}\right|^{2}, \\
& A_{1}\left(U_{0}, U_{1}\right)=2 U_{1}\left|U_{0}\right|^{2}+U_{1}^{*} U_{0}^{2}, \\
& A_{2}\left(U_{0}, U_{1}, U_{2}\right)=2 U_{2}\left|U_{0}\right|^{2}+2\left|U_{1}\right|^{2} U_{0}+U_{2}^{*} U_{0}^{2}+U_{1}^{2} U_{0}^{*}, \\
& A_{3}\left(U_{0}, U_{1}, U_{2}, U_{3}\right)=2 U_{3}\left|U_{0}\right|^{2}+2 U_{2} U_{1}^{*} U_{0}+2 U_{2} U_{0}^{*} U_{1}+2 U_{1} U_{2}^{*} U_{0}+U_{1}\left|U_{1}\right|^{2}+ \\
& U_{0}^{2} U_{3}^{*}, \\
& \ldots
\end{aligned}
$$

and so forth.

With the application of the above recursive relations in equation 222, and using [4, 5, 25, 51, 27] equation 211.

We structure the approximate solutions of $U(z, t)$ as below:

$$
U_{1}(z, t)=C_{1} \mathrm{e}^{j z} \frac{t^{\sigma}}{A \Gamma(\sigma+1)},
$$

with $C_{1}=k \operatorname{sech}^{2}(k z)+(j-B) \tanh (k z)+T_{4} \tanh ^{3}(k z)$,

$$
U_{2}(z, t)=C_{2} \mathrm{e}^{j z} \frac{t^{2 \sigma}}{A^{2} \Gamma(2 \sigma+1)},
$$

... and so forth.

With $C_{2}=2(j-B) k \operatorname{sech}^{2}(k z)+(j-B)^{2} \tanh (k z)+(j-B) T_{4} \tanh ^{2}(k z)+(j-$

185

$2 B) T_{4} \tanh ^{3}(k z)+3 T_{4}^{2} \tanh ^{5}(k z)+\left(2 k T_{4}-2 k^{2}\right) \tanh (k z) \operatorname{sech}^{2}(k z)+3 k T_{4} \tanh ^{2}(k z) \operatorname{sech}^{2}(k z)$.

The approximate solution for the fractional nonlinear Schrödinger equation from equation (5) is obtained by adding all solutions obtained above equation (23), equation (24), and then adding the expression of the initial condition $U_{0}=\tanh (k z) \mathrm{e}^{j z}$ of the dark soliton, we obtain

$$
U_{a p p}(z, t)=U_{0}+U_{1}+U_{2}+\ldots
$$

$$
=\tanh (k z) \mathrm{e}^{j z}+C_{1} \mathrm{e}^{j z} \frac{t^{\sigma}}{A \Gamma(\sigma+1)}+C_{2} \mathrm{e}^{j z} \frac{t^{2 \sigma}}{A^{2} \Gamma(2 \sigma+1)}+\ldots
$$




$$
U_{a p p}(z, t)=\mathrm{e}^{j z}\left\{\tanh (k z)+\frac{C_{1} t^{\sigma}}{A \Gamma(\sigma+1)}+\frac{C_{2} t^{2 \sigma}}{A^{2} \Gamma(2 \sigma+1)}+\ldots\right\},
$$

and the exact solution $U_{\text {exact }}(z, t)$ is obtained when $\alpha \longrightarrow 1$ in equation (25), which means that $\sigma \longrightarrow 2$ because $\sigma=2 \alpha$.

\subsubsection{Fractional bright soliton solutions}

In this subsection, we proceed in the same way as in the previous subsection to point out the behavior of the bright soliton with the effect of the fractional derivative order by Adomian decomposition method. Following the same way in the subsection given the dark soliton above with the same initial condition.

To this end, we structure the approximate solutions of $U(z, t)$ as below:

$$
U_{1}(z, t)=C_{1} \mathrm{e}^{j z} \frac{t^{\sigma}}{A \Gamma(\sigma+1)},
$$

with $C_{1}=(j-B) D \operatorname{sech}(k z)+T_{4} D^{3} \operatorname{sech}^{3}(k z)-D k \tanh (k z) \operatorname{sech}(k z)$,

$$
U_{2}(z, t)=C_{2} \mathrm{e}^{j z} \frac{t^{2 \sigma}}{A^{2} \Gamma(2 \sigma+1)},
$$

... and so forth.

With $C_{2}=(j-B)^{2} D \operatorname{sech}(k z)+\left(T_{4} D^{3}(2 j-3 B)-D k^{2}\right) \operatorname{sech}^{3}(k z)+$

$3 T_{4} D^{5} \operatorname{sech}^{5}(k z)+2(B-j) D k \tanh (k z) \operatorname{sech}(k z)-6 T_{4} D^{3} k \tanh (k z) \operatorname{sech}^{3}(k z)+$ $D k^{2} \tanh ^{2}(k z) \operatorname{sech}(k z)$.

The approximate solution of the Schrödinger fractional nonlinear equation of the equation (5) is obtained by adding all the solutions obtained above the equation (26), the equation 27) and then adding the expression of the initial condition $U_{0}=D \operatorname{sech}(k z) \mathrm{e}^{j z}$ of the bright soliton, we obtain

$$
\begin{gathered}
U_{a p p}(z, t)=U_{0}+U_{1}+U_{2}+\ldots \\
=D \operatorname{sech}(k z) \mathrm{e}^{j z}+C_{1} \mathrm{e}^{j z} \frac{t^{\sigma}}{A \Gamma(\sigma+1)}+C_{2} \mathrm{e}^{j z} \frac{t^{2 \sigma}}{A^{2} \Gamma(2 \sigma+1)}+\ldots
\end{gathered}
$$




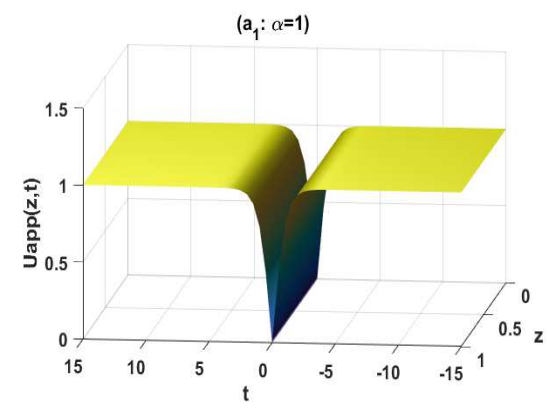

$\left(\mathrm{a}_{3}: \alpha=0.85\right)$

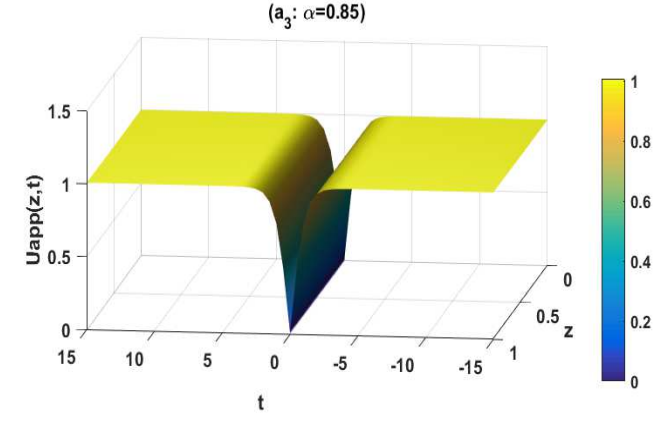

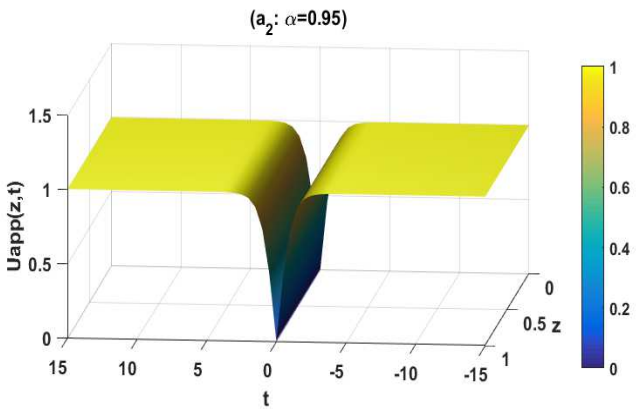

$\left(\mathrm{a}_{4}: \alpha=0.75\right)$

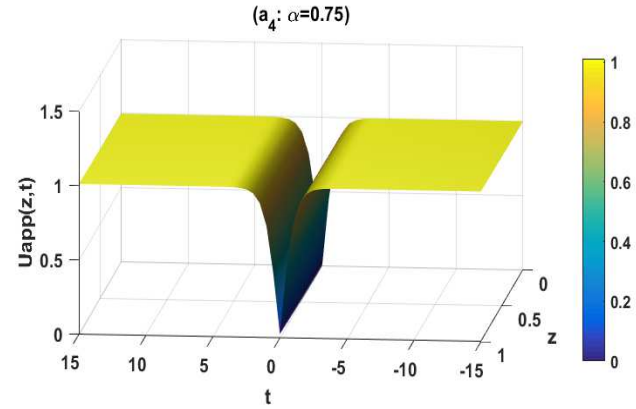

Figure 1: The approximate dark soliton solution obtained by Adomian decomposition method of the equation 25 when $(\alpha=1, \alpha=0.95, \alpha=0.85, \alpha=0.75), \omega_{0}=0.032$ rad.s ${ }^{-1}, C_{0}=$ $100.045 p H, C_{L}=78.65 p H, L_{L}=200.054 n H, \rho=0.01, k=0.98$.
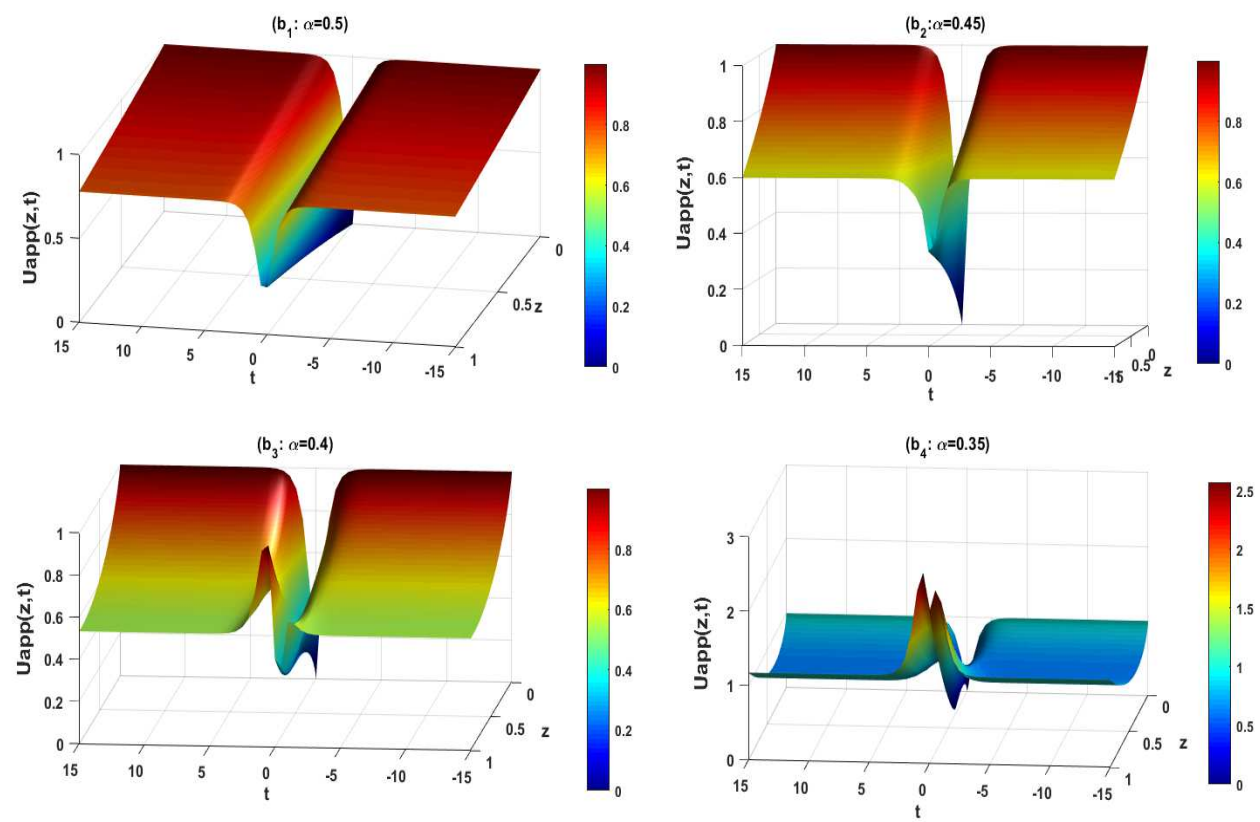

12

Figure 2: The approximate dark soliton solution obtained by Adomian decomposition method of the equation 25 when $(\alpha=0.5, \alpha=0.45, \alpha=0.4, \alpha=0.35), \omega_{0}=0.032$ rad. $s^{-1}$, $C_{0}=100.045 p H, C_{L}=78.65 p H, L_{L}=200.054 n H, \rho=0.01, k=0.98$. 

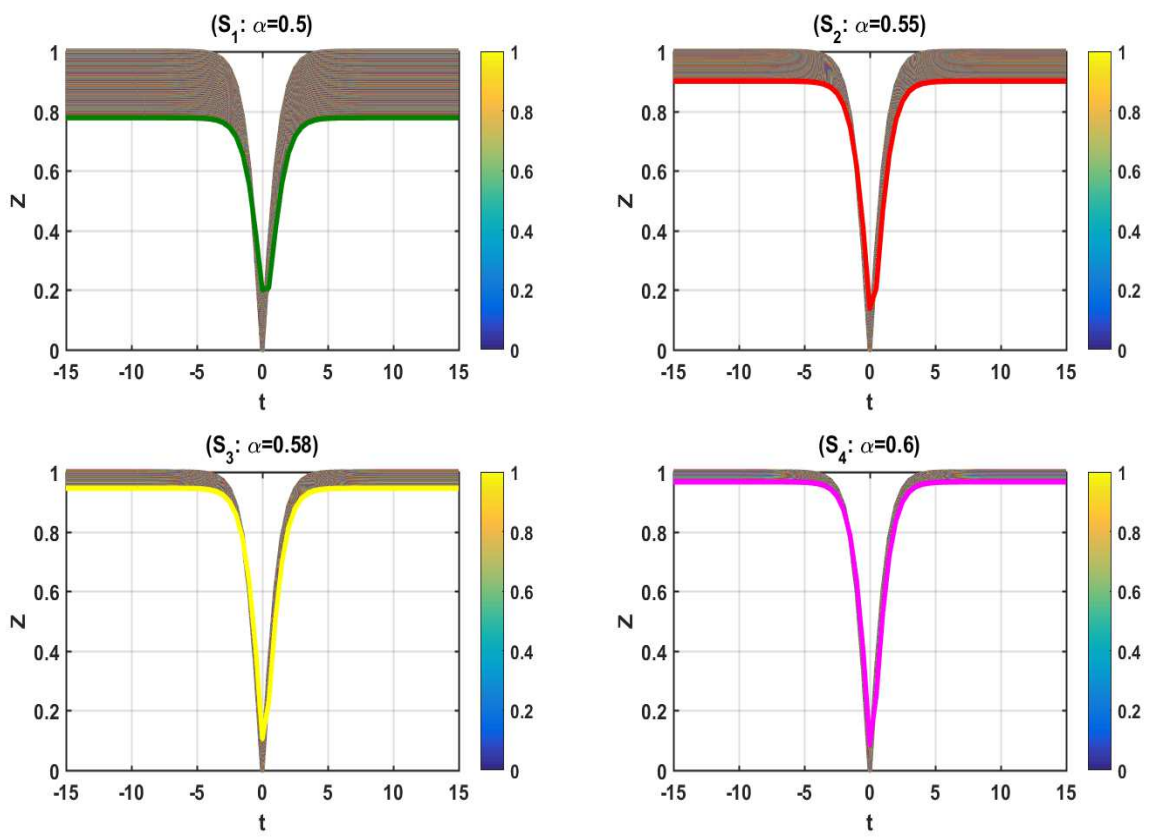

Figure 3: The approximate dark soliton solution obtained by Adomian decomposition method of the equation 25 when $(\alpha=0.5, \alpha=0.55, \alpha=0.58, \alpha=0.6), \omega_{0}=0.032 \mathrm{rad} . \mathrm{s}^{-1}$, $C_{0}=100.045 p H, C_{L}=78.65 p H, L_{L}=200.054 n H, \rho=0.01, k=0.7$. 


$$
U_{a p p}(z, t)=\mathrm{e}^{j z}\left\{D \operatorname{sech}(k z)+\frac{C_{1} t^{\sigma}}{A \Gamma(\sigma+1)}+\frac{C_{2} t^{2 \sigma}}{A^{2} \Gamma(2 \sigma+1)}+\ldots\right\},
$$

and the exact solution $U_{\text {exact }}(z, t)$ is obtained when $\alpha \longrightarrow 1$ in equation (28), which means that $\sigma \longrightarrow 2$ because $\sigma=2 \alpha$.

\subsection{Modulation instability gain}

The modulation instability (MI) is the one important phenomenon which happens when the nonlinear and dispersion terms are present in a nonlinear evolution equation. Usually modulation instability is present in a nonlinear system because of the low perturbations enforce on the continuous wave. Now, we consider the steady-state solution of equation (1) as follows

$$
U(z, t)=\sqrt{P} \mathrm{e}^{j T_{4} P z},
$$

where $P$ is the power incident. Suppose the perturb solution of equation (1) in the following expression

$$
U(z, t)=(\sqrt{P}+\varepsilon(z, t)) \mathrm{e}^{j T_{4} P z} .
$$

Taking in to account the impact of the perturb field, we obtain the linearized equation

$$
\frac{\partial \varepsilon(\mathrm{z}, \mathrm{t})}{\partial z}-\beta_{2} \frac{j^{-3 \alpha}}{(2 \alpha) !} \frac{\partial^{2 \alpha} \varepsilon(\mathrm{z}, \mathrm{t})}{\partial t^{2 \alpha}}-2 \beta_{2} \frac{j^{-2 \alpha}}{(2 \alpha) !} \frac{\partial^{\alpha} \varepsilon(\mathrm{z}, \mathrm{t})}{\partial t^{\alpha}}+T_{4} P\left(\varepsilon(z, t)+\varepsilon^{*}(z, t)\right)=0 .
$$

Where $\varepsilon^{*}(z, t)$ is the complex conjugate of $\varepsilon(z, t)$. Now we suppose the solution for the perturbed expression as follows

$$
\varepsilon(z, t)=a_{1} e^{j(K z+\Omega t)}+a_{2} e^{-j(K z+\Omega t)},
$$

where $a_{1}$ and $a_{2}$ are reals, while $K$ and $\Omega$ are the wave number and modulation frequency, respectively. Let's now introduce equation 32 into equation (31) gives 

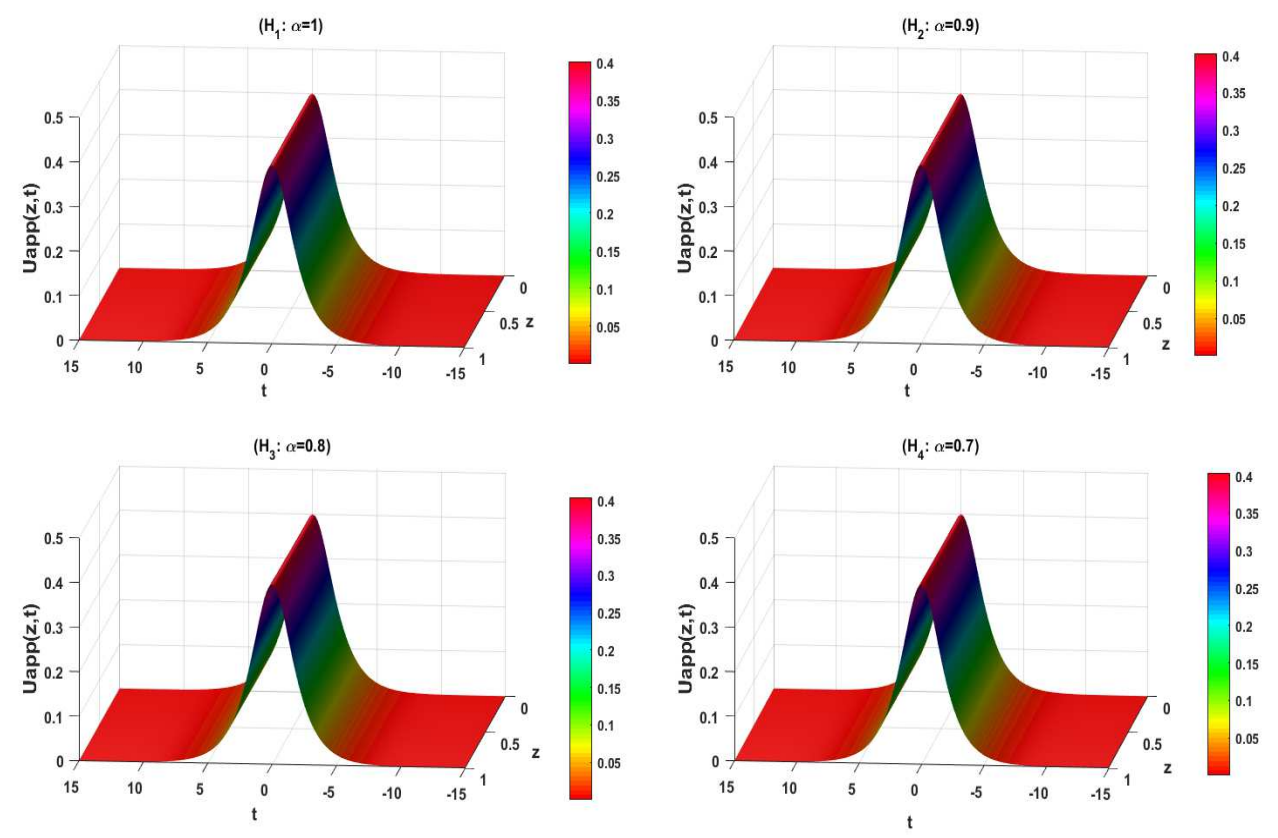

Figure 4: The approximate bright soliton solution obtained by Adomian decomposition method of the equation 28 when $(\alpha=1, \alpha=0.9, \alpha=0.8, \alpha=0.7), \omega_{0}=0.032 \mathrm{rad}_{\mathrm{s}} \mathrm{s}^{-1}, C_{0}=$ $100.045 p H, C_{L}=78.65 p H, L_{L}=200.054 n H, \rho=0.01, k=0.7, D=0.084$.
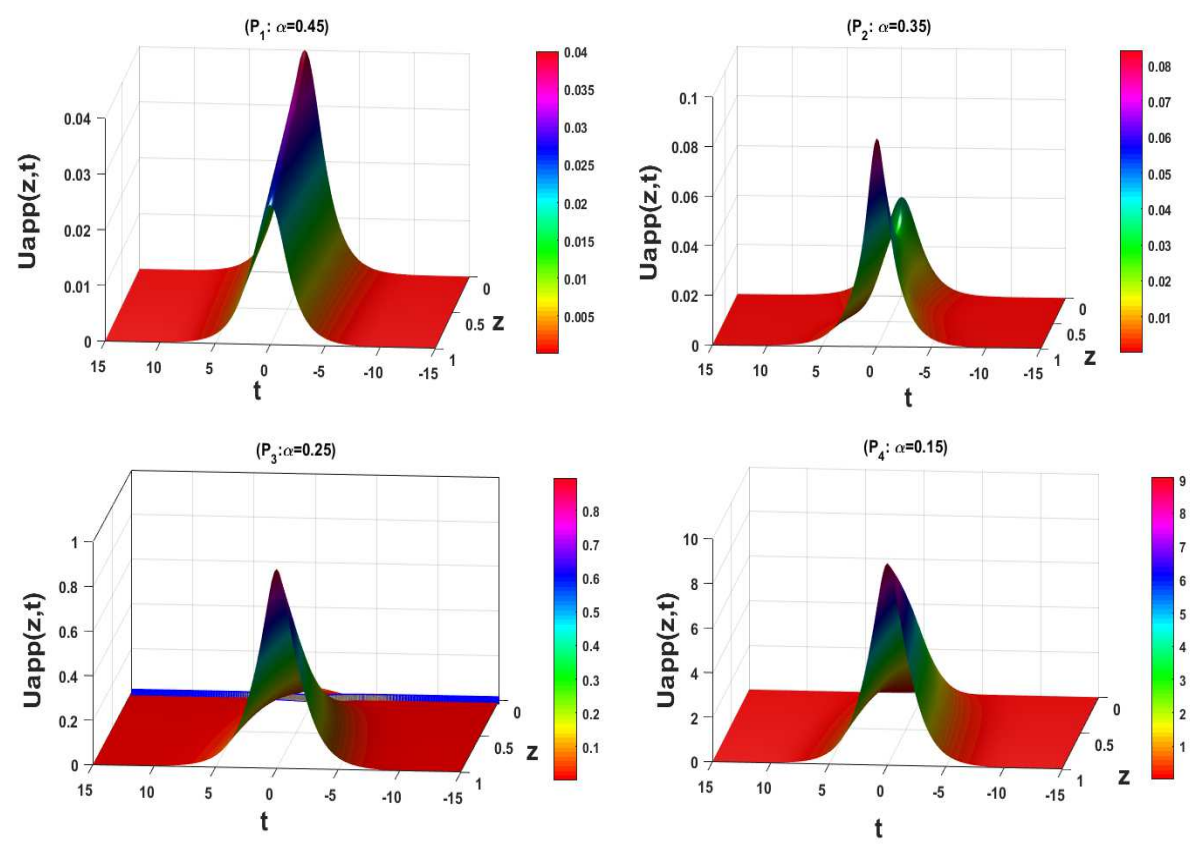

15

Figure 5: The approximate bright soliton solution obtained by Adomian decomposition method of the equation 28 when $(\alpha=0.45, \alpha=0.35, \alpha=0.25, \alpha=0.15), \omega_{0}=0.032 \mathrm{rad} . \mathrm{s}^{-1}$, $C_{0}=100.045 \mathrm{pH}, C_{L}=78.65 \mathrm{pH}, L_{L}=200.054 \mathrm{nH}, \rho=0.01, k=0.7, D=0.084$. 

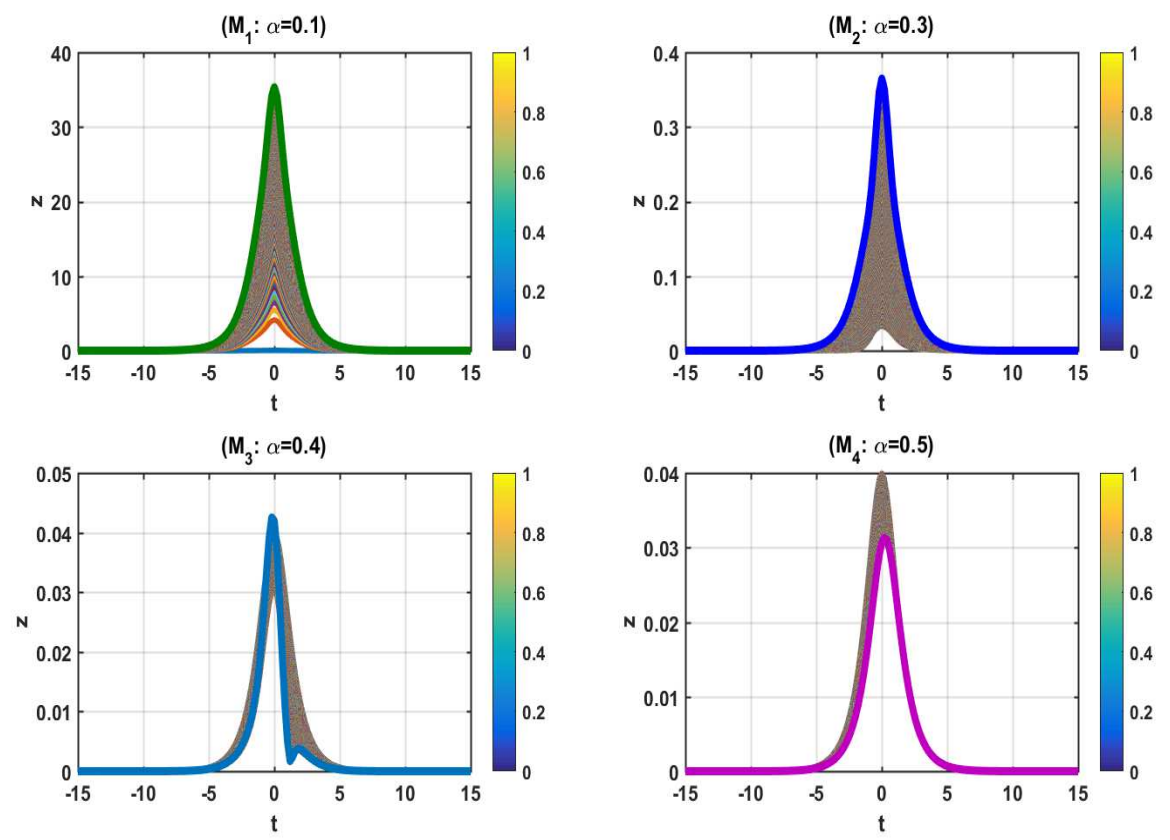

Figure 6: The approximate bright soliton solution obtained by Adomian decomposition method of the equation 28 when $(\alpha=0.1, \alpha=0.3, \alpha=0.4, \alpha=0.5), \omega_{0}=0.032 \mathrm{rad} . \mathrm{s}^{-1}$, $C_{0}=100.045 p H, C_{L}=78.65 p H, L_{L}=200.054 n H, \rho=0.01, k=0.95, D=0.084$. 
Table 1: Comparison between the absolute value of the approximate dark soliton solution obtained by Adomian decomposition method of the equation 25 and the absolute value of the exact solution when $(\alpha=1), \omega_{0}=0.032 \mathrm{rad} . \mathrm{s}^{-1}, C_{0}=500.045 \mathrm{pH}, C_{L}=478.65 \mathrm{pH}$, $L_{L}=200.0540 n H, \rho=0.01, k=0.75$.

\begin{tabular}{llll}
$\mathrm{N}=1,2, \ldots 10$ & $\operatorname{abs}\left(\mathrm{U}_{\text {app }}\right)$ & $\operatorname{abs}\left(\mathrm{U}_{\text {exact }}\right)$ & $\operatorname{Error}\left|\left(U_{\text {app }}\right)-\left(U_{\text {exact }}\right)\right|$ \\
\hline 1 & 0.7531 & 0.9951 & 0.2420 \\
2 & 0.7531 & 0.9951 & 0.2420 \\
3 & 0.7531 & 0.9951 & 0.2420 \\
4 & 0.7531 & 0.9951 & 0.2420 \\
5 & 0.7531 & 0.9951 & 0.2420 \\
6 & 0.7531 & 0.9951 & 0.2420 \\
7 & 0.7531 & 0.9951 & 0.2420 \\
8 & 0.7531 & 0.9951 & 0.2420 \\
9 & 0.7531 & 0.9951 & 0.2420 \\
10 & 0.7531 & 0.9951 & 0.2420 \\
\hline
\end{tabular}

$$
\left(\begin{array}{cc}
j K-(\Omega)^{2 \alpha} \beta_{2} \frac{j^{-\alpha}}{(2 \alpha) !}-2 \beta_{2}(\Omega)^{\alpha} \frac{j^{-\alpha}}{(2 \alpha) !} & 2 T_{4} P \\
2 T_{4} P & -j K-(-\Omega)^{2 \alpha} \beta_{2} \frac{j^{-5 \alpha}}{(2 \alpha) !}-2(-\Omega)^{\alpha} \frac{j^{-3 \alpha}}{(2 \alpha) !}
\end{array}\right)\left(\begin{array}{l}
a_{1} \\
a_{2}
\end{array}\right)=\left(\begin{array}{l}
0 \\
0
\end{array}\right)
$$

the obtained $2 \times 2$ matrix has a non trivial solution when the determinant vanishes. So, the determinant linked with this matrix can read as

$$
K^{2}+B-4 T_{4}^{2} P^{2}=0
$$

and we show that the general solution is valid unless :

$$
K= \pm 2 T_{4} P \sqrt{1-\frac{B}{4 P^{2} T_{4}^{2}}}
$$

with $B=\left((\Omega)^{2 \alpha} \beta_{2} \frac{j^{-\alpha}}{(2 \alpha) !}+2 \beta_{2}(\Omega)^{\alpha} \frac{j^{-\alpha}}{(2 \alpha) !}\right)\left((-\Omega)^{2 \alpha} \beta_{2} \frac{j^{-5 \alpha}}{(2 \alpha) !}+2 \beta_{2}(-\Omega)^{\alpha} \frac{j^{-3 \alpha}}{(2 \alpha) !}\right)$. 

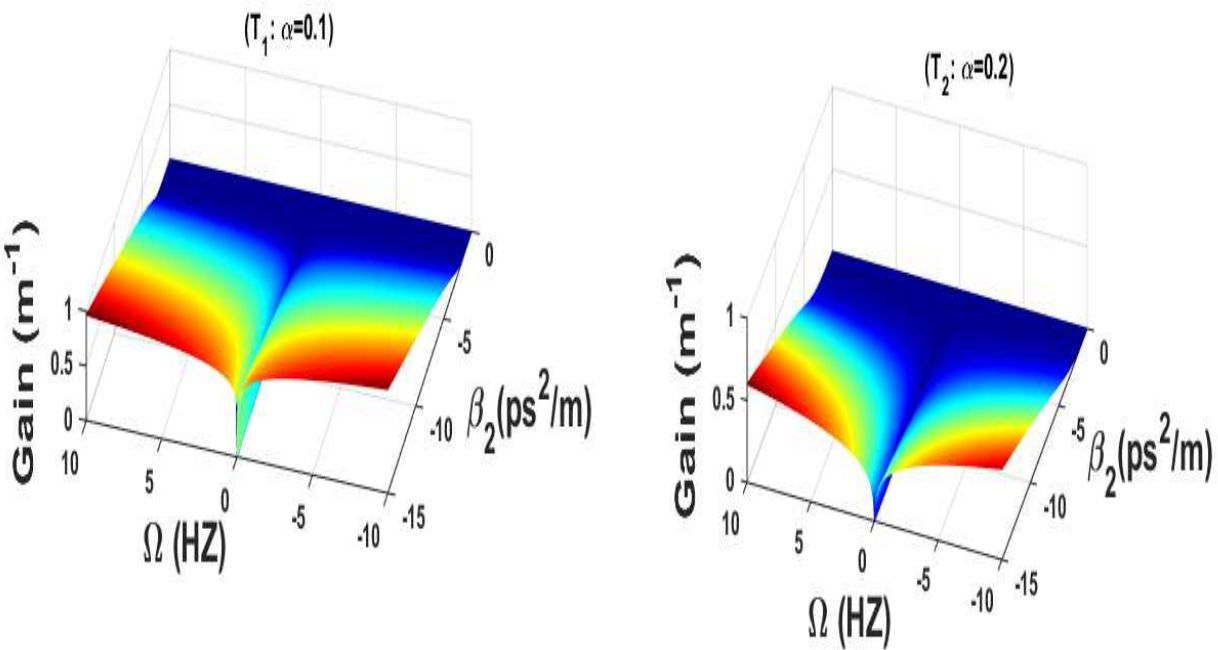

Figure 7: The variation of the (MI) Gain spectrum versus Group velocity dispersion of the

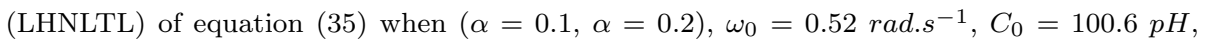
$C_{L}=500.65 \mathrm{pH}, L_{L}=400.2 \mathrm{nH}, \rho=20.02, P=120$.
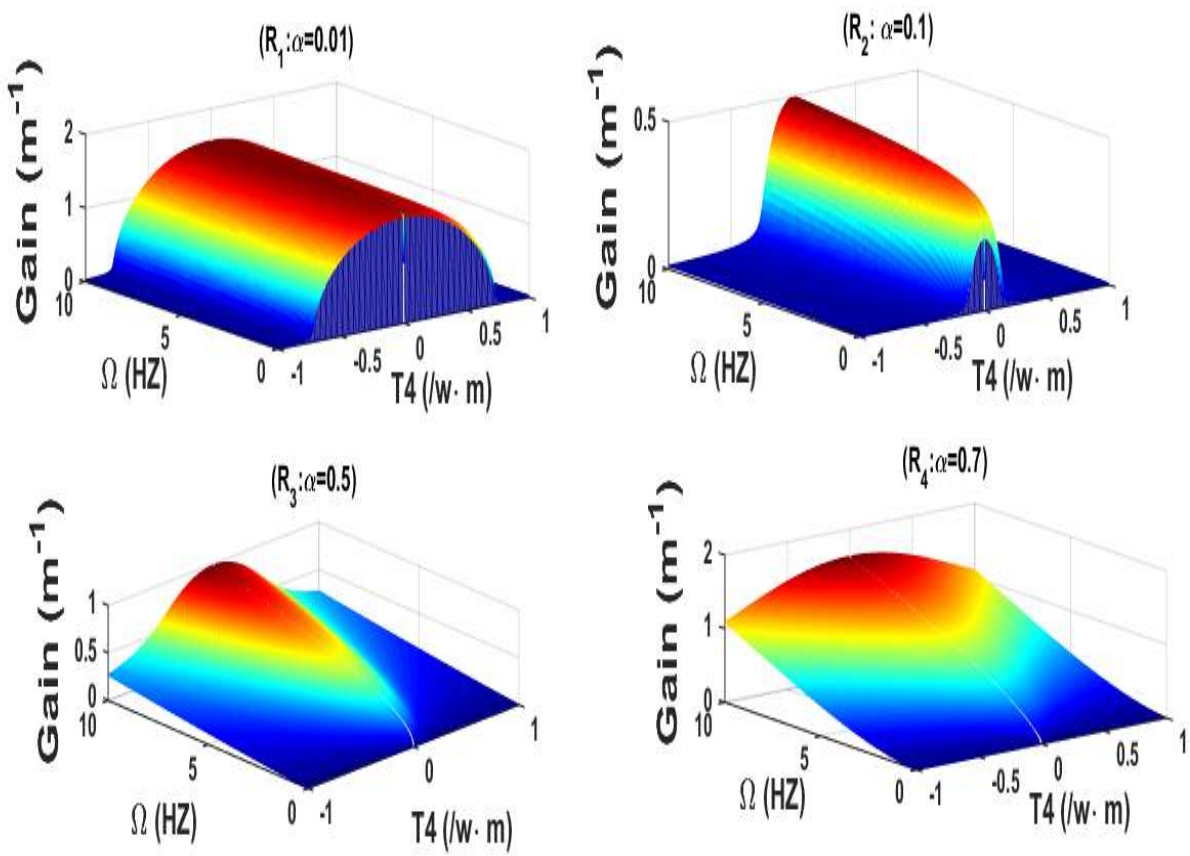

18

Figure 8: The variation of the (MI) Gain spectrum versus the kerr nonlinearty of the (LHNLTL) of equation (35) when $(\alpha=0.01, \alpha=0.1, \alpha=0.5, \alpha=0.7), \omega_{0}=0.32 \mathrm{rad}_{\mathrm{s}} \mathrm{s}^{-1}$, $C_{0}=70.6 \mathrm{pH}, C_{L}=450.65 \mathrm{pH}, L_{L}=420.2 \mathrm{nH}, \rho=0.002, P=0.5$. 
Table 2: Comparison between the absolute value of the approximate dark soliton solution obtained by Adomian decomposition method of the equation 25 and the absolute value of

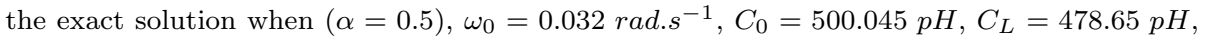
$L_{L}=200.0540 n H, \rho=0.01, k=0.75$.

\begin{tabular}{llll}
$\mathrm{N}=1,2, \ldots 10$ & $\operatorname{abs}\left(\mathrm{U}_{\text {app }}\right)$ & $\operatorname{abs}\left(\mathrm{U}_{\text {exact }}\right)$ & Error $\left|\left(U_{\text {app }}\right)-\left(U_{\text {exact }}\right)\right|$ \\
\hline 1 & 0.7531 & 0.9951 & 0.2420 \\
2 & 0.7528 & 0.9920 & 0.2392 \\
3 & 0.7526 & 0.9890 & 0.2364 \\
4 & 0.7523 & 0.9860 & 0.2337 \\
5 & 0.7520 & 0.9830 & 0.2310 \\
6 & 0.7517 & 0.9800 & 0.2282 \\
7 & 0.7514 & 0.9770 & 0.2256 \\
8 & 0.7511 & 0.9740 & 0.2229 \\
9 & 0.7508 & 0.9710 & 0.2203 \\
10 & 0.7504 & 0.9681 & 0.2177
\end{tabular}

\section{Physical explanation of the results}

Figures 1 and 2 show the pipe of the dark soliton solutions with the effect of the fractional derivative order in nonlinear left-handed transmission line for $\omega_{0}=0.032 \mathrm{rad} . \mathrm{s}^{-1}, C_{0}=100.045 \mathrm{pH}, C_{L}=78.65 \mathrm{pH}, L_{L}=200.054 \mathrm{nH}$, $\rho=0.01, k=0.98$. The effect of the fractional order are pointed out when $\alpha=0.5, \alpha=0.45, \alpha=0.4$ and $\alpha=0.35$ (see figure 2). In addition the shape of the wave undergoes deformations as $\alpha$ takes small values and this reflects the phenomenon of the memory effect which is one of the advantages linked to the fractional derivative order. On the other hand, for values of $\alpha$ tending towards 1 , the result obtained is similar to the dark soliton solution known in the left-handed metamaterials. We also emphasized the behavior of 
Table 3: Comparison between the absolute value of the approximate dark soliton solution obtained by Adomian decomposition method of the equation 25 and the absolute value of

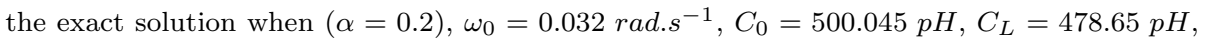
$L_{L}=200.0540 n H, \rho=0.01, k=0.75$.

\begin{tabular}{llll}
$\mathrm{N}=1,2, \ldots 10$ & $\operatorname{abs}\left(\mathrm{U}_{\text {app }}\right)$ & abs $\left(\mathrm{U}_{\text {exact }}\right)$ & Error $\left|\left(U_{\text {app }}\right)-\left(U_{\text {exact }}\right)\right|$ \\
\hline 1 & 0.7531 & 0.9951 & 0.2420 \\
2 & 2.4983 & 3.1348 & 0.6365 \\
3 & 4.7712 & 6.0274 & 1.2562 \\
4 & 6.9457 & 8.7256 & 1.7799 \\
5 & 9.0417 & 11.2942 & 2.2526 \\
6 & 11.0746 & 13.7666 & 2.6920 \\
7 & 13.0553 & 16.1628 & 3.1075 \\
8 & 14.9918 & 18.4963 & 3.5044 \\
9 & 16.8900 & 20.7764 & 3.8865 \\
10 & 18.7544 & 23.0105 & 4.2561 \\
\hline
\end{tabular}

the bright soliton solutions with the effect of the fractional derivative order

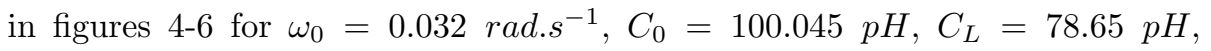
$L_{L}=200.054 n H, \rho=0.01, k=0.7, k=0.95, D=0.084$. Moreover, the effect of fractional order on the width of the bright soliton is obtained (see Figures 5 and 6 ). More precisely, the solitons obtained took into account the terms of nonlinearity and of dispersion for their existence. As it is mentioned in several works that the nonlinearity and dispersion terms involve the modulation instability, we have set out the dynamic of the variation of MI gain spectra in the anomalous group velocity dispersion on the one hand and on the other hand the nonlinear term kerr. It is observed the instability zones when the derivative order $\alpha$ is near to 1 (see figures $8\left(R_{3}, R_{4}\right)$ ). It should be noted that the instability is linked to MI due to unbidden temporal modulation of the continuous wave (CW) stack and change it into a pulse train. However, for the small value of the fractional derivative order stability zones appeared (see 
Table 4: Comparison between the absolute value of the approximate bright soliton solution obtained by Adomian decomposition method of the equation 28 and the absolute value of

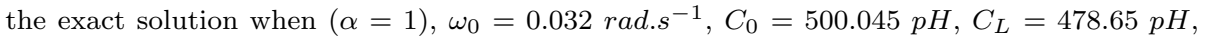
$L_{L}=200.0540 n H, \rho=0.01, k=0.75$.

\begin{tabular}{llll}
$\mathrm{N}=1,2, \ldots 10$ & $\operatorname{abs}\left(\mathrm{U}_{\text {app }}\right)$ & $\operatorname{abs}\left(\mathrm{U}_{\text {exact }}\right)$ & Error $\left|\left(U_{\text {app }}\right)-\left(U_{\text {exact }}\right)\right|$ \\
\hline 1 & 0.0038 & 0.0039 & $0.1622 \mathrm{e}-03$ \\
2 & 0.0038 & 0.0039 & $0.1622 \mathrm{e}-03$ \\
3 & 0.0038 & 0.0039 & $0.1622 \mathrm{e}-03$ \\
4 & 0.0038 & 0.0039 & $0.1622 \mathrm{e}-03$ \\
5 & 0.0038 & 0.0039 & $0.1622 \mathrm{e}-03$ \\
6 & 0.0038 & 0.0039 & $0.1622 \mathrm{e}-03$ \\
7 & 0.0038 & 0.0039 & $0.1622 \mathrm{e}-03$ \\
8 & 0.0038 & 0.0039 & $0.1622 \mathrm{e}-03$ \\
9 & 0.0038 & 0.0039 & $0.1622 \mathrm{e}-03$ \\
10 & 0.0038 & 0.0039 & $0.1622 \mathrm{e}-03$ \\
\hline
\end{tabular}

figures $\left.8\left(R_{1}, R_{2}\right)\right)$. Enough but not the last, we use MATLAB calculation to dig out the gap between the approximate analytical results and the exacts. From tables 1 and 4 it is observed that when the value of derivative order tends to 1 the $U_{a p p} \approx U_{\text {exact }}$. It is also observed that the difference is greater between the exact obtained and the results of the estimation when the values of the order of the derivatives are too small (see tables $2,3,5$ and 6 ). So, these results obtained are new and significant because dark and bright solitons were obtained by the adomian decomposition method in a nonlinear left-handed transmission line with the variable $(t)$ less than, greater than or equal to zero. We deduce that the behavior of the approximate bright solutions is practically the same as the behavior of its exact solution with different values of $\alpha$ and we obtain small errors between the approximate solution and the exact solution for certain values of $\alpha$ with the number of steps. On the other hand, the behavior of dark approximate solutions and the behavior of its exact solution, leads us to large 
Table 5: Comparison between the absolute value of the approximate bright soliton solution obtained by Adomian decomposition method of the equation 28 and the absolute value of

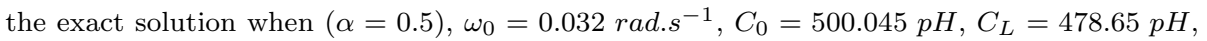
$L_{L}=200.0540 n H, \rho=0.01, k=0.75$.

\begin{tabular}{llll}
$\mathrm{N}=1,2, \ldots 10$ & $\operatorname{abs}\left(\mathrm{U}_{\text {app }}\right)$ & $\operatorname{abs}\left(\mathrm{U}_{\text {exact }}\right)$ & $\operatorname{Error}\left|\left(U_{\text {app }}\right)-\left(U_{\text {exact }}\right)\right|$ \\
\hline 1 & 0.0038 & 0.0039 & $0.1622 \mathrm{e}-03$ \\
2 & 0.0038 & 0.0039 & $0.1622 \mathrm{e}-03$ \\
3 & 0.0037 & 0.0039 & $0.1622 \mathrm{e}-03$ \\
4 & 0.0037 & 0.0039 & $0.1623 \mathrm{e}-03$ \\
5 & 0.0037 & 0.0039 & $0.1623 \mathrm{e}-03$ \\
6 & 0.0037 & 0.0038 & $0.1623 \mathrm{e}-03$ \\
7 & 0.0037 & 0.0038 & $0.1623 \mathrm{e}-03$ \\
8 & 0.0036 & 0.0038 & $0.1623 \mathrm{e}-03$ \\
9 & 0.0036 & 0.0038 & $0.1623 \mathrm{e}-03$ \\
10 & 0.0036 & 0.0038 & $0.1623 \mathrm{e}-03$ \\
\hline
\end{tabular}

errors for some $\alpha$ values with the number of steps. Therefore, we infer that the bright soliton is more convergent than the dark soliton. This work could therefore be very interesting.

\section{Summary} cessfully applied to obtain semi-analytic solutions of the fractional nonlinear Schrödinger equation with initial conditions. Efficiency, power, reliability and reduced calculations of this approach give it a wider applicability. Figures 1, 2 and 3 show the behavior of the dark soliton with the influence of the fractional tained with deeply small values of the derivative order. Concerning the bright soliton solutions it is also pointed out the same phenomenon observed previously to the dark solitons. As mentioned above, the divergence between kerr nonlin- 
Table 6: Comparison between the absolute value of the approximate bright soliton solution obtained by Adomian decomposition method of the equation 28 and the absolute value of

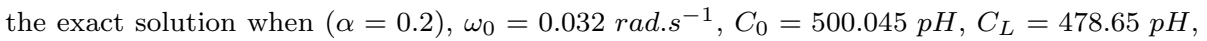
$L_{L}=200.0540 n H, \rho=0.01, k=0.75$.

\begin{tabular}{llll}
$\mathrm{N}=1,2, \ldots 10$ & $\operatorname{abs}\left(\mathrm{U}_{\text {app }}\right)$ & $\operatorname{abs}\left(\mathrm{U}_{\text {exact }}\right)$ & Error $\left|\left(U_{\text {app }}\right)-\left(U_{\text {exact }}\right)\right|$ \\
\hline 1 & 0.0038 & 0.0039 & 0.0002 \\
2 & 0.0236 & 0.0236 & 0.0001 \\
3 & 0.0438 & 0.0438 & 0.0000 \\
4 & 0.0620 & 0.0621 & 0.0002 \\
5 & 0.0789 & 0.0793 & 0.0004 \\
6 & 0.0951 & 0.0957 & 0.0007 \\
7 & 0.1106 & 0.1116 & 0.0010 \\
8 & 0.1256 & 0.1269 & 0.0013 \\
9 & 0.1401 & 0.1418 & 0.0016 \\
10 & 0.1543 & 0.1563 & 0.0020 \\
\hline
\end{tabular}

earity and group velocity terms is favorable to MI, then the MI gain spectrum versus anomalous group velocity dispersion and nonlinearity coefficient is also shown (see figures 7 and 8 ). Several instability zones are obtained under the effect of the derivative order. Beside these results, we obtain the errors between the approximate and the exacts dark (bright) solitons solutions (see tables 1 , $2,3,4,5$ and 6$)$. Therefore, we deduce that the approximate solution is convergent series as an exact solution [27. The Adomian decomposition method is a very efficient and powerful technique for finding soliton solutions. However, interpretation of the results reveals that the propagation of the wave is considerably disturbed when moving away with the effect of the derivative order, so that the evolution of the system can no longer be controlled. Nevertheless, these results are more precise than that in [39, 40, because in this work, we obtain both dark and bright solitons with the effect of fractional derivatives order by applying the Adomian decomposition method (ADM) to this same equation (1) 
of [39, 40] and we also evaluated the modulational instability of these solitons. Since, analytical and numerical studies of the metamaterial transmission line have proven the presence of Schrödinger solitons, which has led us to formation of dark Schrödinger solitons in this nonlinear left-handed transmission line by applying the Adomian decomposition method (ADM) to the equation (1) of [39, 40] with the effect of fractional derivatives order. Finally, this nonlinear left-handed transmission line with the effect of the fractional derivative order also allowed us to generate bright envelope solitons by making an appropriate choice of the values of the fractional derivative order and the initial condition.

\section{Author contributions}

All authors contributed significantly to this article and have read and approved the final manuscript.

\section{Financial disclosure}

This research did not receive any external funding.

\section{Declaration of Competing Interest}

The authors declare that they have no known competing financial interests or personal relationships that could have appeared to disrupt the work done in this article.

\section{Acknowledgments}

We did not receive any funding from any organization for this research. 


\section{References}

325 [1] M. El amine CHAIB, Modélisation et caractérisation de fonctions électroniques générées par des dispositifs à métamatériaux, Mémoire de Magistère en Systèmes des Réseaux de Télécommunication. Université ABOU BEKR BELKAID TLEMCEN (2012).

[2] V. G. Veselago, The electrodynamics of substances with simultaneously negative values of img align $=$ absmiddle alt $=\mathrm{eps} / \mathrm{img}$ and $\mu$, PhysicsUspekhi 10 (4) (1968) 509-514.

[3] J. B. Pendry, A. J. Holden, D. J. Robbins, W. Stewart, et al., Magnetism from conductors and enhanced nonlinear phenomena, IEEE transactions on microwave theory and techniques 47 (11) (1999) 2075-2084.

[4] A. A. A. Kilbas, H. M. Srivastava, J. J. Trujillo, Theory and applications of fractional differential equations, Vol. 204, Elsevier Science Limited, 2006.

[5] I. Podlubny, Fractional differential equations, vol. 198 of mathematics in science and engineering (1999).

[6] S. G. Samko, A. A. Kilbas, O. I. Marichev, et al., Fractional integrals and derivatives, Vol. 1993, Gordon and Breach Science Publishers, Yverdon Yverdon-les-Bains, Switzerland, 1993.

[7] A. M. El-Sayed, Fractional-order diffusion-wave equation, International Journal of Theoretical Physics 35 (2) (1996) 311-322.

[8] M. A. Herzallah, A. M. El-Sayed, D. Baleanu, On the fractional-order diffusion-wave process, Rom. J. Phys 55 (3-4) (2010) 274-284.

[9] M. A. Herzallah, S. I. Muslih, D. Baleanu, E. M. Rabei, Hamilton-jacobi and fractional like action with time scaling, Nonlinear Dynamics 66 (4) (2011) 549-555.

[10] R. L. Magin, Fractional calculus in bioengineering, Begell House Redding, 2006. 
[11] B. West, M. Bologna, P. Grigolini, Institute for nonlinear science, in: Physics of Fractal Operators, Springer, 2003.

[12] I. S. Jesus, J. T. Machado, Fractional control of heat diffusion systems, Nonlinear Dynamics 54 (3) (2008) 263-282.

[13] O. P. Agrawal, D. Baleanu, A hamiltonian formulation and a direct numerical scheme for fractional optimal control problems, Journal of Vibration and Control 13 (9-10) (2007) 1269-1281.

[14] V. E. Tarasov, Fractional vector calculus and fractional maxwell's equations, Annals of Physics 323 (11) (2008) 2756-2778.

[15] S.-f. Deng, Bäcklund transformation and soliton solutions for kp equation, Chaos, Solitons \& Fractals 25 (2) (2005) 475-480.

[16] O. Pashaev, G. Tanoğlu, Vector shock soliton and the hirota bilinear method, Chaos, Solitons \& Fractals 26 (1) (2005) 95-105.

[17] V. Vakhnenko, E. Parkes, A. Morrison, A bäcklund transformation and the inverse scattering transform method for the generalised vakhnenko equation, Chaos, Solitons \& Fractals 17 (4) (2003) 683-692.

[18] L. De-Sheng, G. Feng, Z. Hong-Qing, Solving the (2+ 1)-dimensional higher order broer-kaup system via a transformation and tanh-function method, Chaos, Solitons \& Fractals 20 (5) (2004) 1021-1025.

[19] T. A. Abassy, M. A. El-Tawil, H. K. Saleh, The solution of kdv and mkdv equations using adomian pade approximation, International Journal of Nonlinear Sciences and Numerical Simulation 5 (4) (2004) 327-340.

[20] H.-M. Liu, Generalized variational principles for ion acoustic plasma waves by he's semi-inverse method, Chaos, Solitons \& Fractals 23 (2) (2005) 573576. 
[21] J.-H. He, Variational iteration method-a kind of non-linear analytical technique: some examples, International journal of non-linear mechanics 34 (4) (1999) 699-708.

[22] J.-H. He, Modified lindstedt-poincare methods for some strongly non-linear oscillations: Part i: expansion of a constant, International Journal of NonLinear Mechanics 37 (2) (2002) 309-314.

[23] A.-M. Wazwaz, A reliable technique for solving the wave equation in an infinite one-dimensional medium, Applied Mathematics and Computation 92 (1) (1998) 1-7.

[24] E. Zayed, T. Nofal, K. Gepreel, Homotopy perturbation and adomain decomposition methods for solving nonlinear boussinesq equations, Communications on Applied Nonlinear Analysis 15 (3) (2008) 57.

[25] G. Adomian, Solving frontier problems of physics: the decomposition method, Vol. 60, Springer Science \& Business Media, 2013.

[26] G. Adomian, Stochastic systems analysis, in: Applied Stochastic Processes, Elsevier, 1980, pp. 1-17.

[27] S. Ray, Nonlinear Differential Equations in Physics, Springer, 2020.

[28] D. Wang, H.-Q. Zhang, Further improved f-expansion method and new exact solutions of konopelchenko-dubrovsky equation, Chaos, Solitons \& Fractals 25 (3) (2005) 601-610.

[29] X.-H. B. Wu, J.-H. He, Solitary solutions, periodic solutions and compacton-like solutions using the exp-function method, Computers \& Mathematics with Applications 54 (7-8) (2007) 966-986.

[30] J.-H. He, Homotopy perturbation method for solving boundary value problems, Physics letters A 350 (1-2) (2006) 87-88. 
[31] K. A. Gepreel, The homotopy perturbation method applied to the nonlinear fractional kolmogorov-petrovskii-piskunov equations, Applied Mathematics Letters 24 (8) (2011) 1428-1434.

[32] Z.-Z. Lan, J.-J. Su, Solitary and rogue waves with controllable backgrounds for the non-autonomous generalized ab system, Nonlinear Dynamics 96 (4) (2019) 2535-2546.

[33] Z.-Z. Lan, W.-Q. Hu, B.-L. Guo, General propagation lattice boltzmann model for a variable-coefficient compound kdv-burgers equation, Applied Mathematical Modelling 73 (2019) 695-714.

[34] Z. Lan, Periodic, breather and rogue wave solutions for a generalized (3+ 1)-dimensional variable-coefficient b-type kadomtsev-petviashvili equation in fluid dynamics, Applied Mathematics Letters 94 (2019) 126-132.

[35] Z.-Z. Lan, Rogue wave solutions for a coupled nonlinear schrödinger equation in the birefringent optical fiber, Applied Mathematics Letters 98 (2019) $128-134$.

[36] Z. Lan, Soliton and breather solutions for a fifth-order variable-coefficient nonlinear schrödinger equation in an optical fiber, Applied Mathematics Letters 102 (2020) 106132.

[37] Z.-Z. Lan, B.-L. Guo, Nonlinear waves behaviors for a coupled generalized nonlinear schrodinger-boussinesq system in a homogeneous magnetized plasma, NONLINEAR DYNAMICS 100 (4) (2020) 3771-3784.

[38] Z.-Z. Lan, Pfaffian and extended pfaffian solutions for a (3+ 1)-dimensional generalized wave equation, Physica Scripta 94 (12) (2019) 125221.

[39] D. Ahmadou, M. Justin, B. M. Hubert, G. Betchewe, D. Y. Serge, K. T. Crépin, Dark solitons and modulational instability of the nonlinear lefthanded transmission electrical line with fractional derivative order, Physica Scripta 95 (10) (2020) 105803. 
[40] S. Abdoulkary, A. D. Aboubakar, M. Aboubakar, A. Mohamadou, L. Kavitha, Solitary wave solutions and modulational instability analysis of the nonlinear schrödinger equation with higher-order nonlinear terms in the left-handed nonlinear transmission lines, Communications in Nonlinear Science and Numerical Simulation 22 (1-3) (2015) 1288-1296.

[41] S. Nestor, A. Houwe, G. Betchewe, S. Y. Doka, et al., A series of abundant new optical solitons to the conformable space-time fractional perturbed nonlinear schrödinger equation, Physica Scripta 95 (8) (2020) 085108.

[42] C. Li, D. Qian, Y. Chen, On riemann-liouville and caputo derivatives, Discrete Dynamics in Nature and Society 2011 (2011).

[43] R. Khalil, M. Al Horani, A. Yousef, M. Sababheh, A new definition of fractional derivative, Journal of Computational and Applied Mathematics 264 (2014) 65-70.

[44] A. R. Kanth, K. Aruna, Two-dimensional differential transform method for solving linear and non-linear schrödinger equations, Chaos, Solitons \& Fractals 41 (5) (2009) 2277-2281.

[45] E. P. Gross, Hydrodynamics of a superfluid condensate, Journal of Mathematical Physics 4 (2) (1963) 195-207.

[46] K. B. Dysthe, Modelling a" rogue wave"-speculations or a realistic possibility, Rogues Waves 2000 (2001) 255-264.

[47] K. Henderson, D. Peregrine, J. Dold, Unsteady water wave modulations: fully nonlinear solutions and comparison with the nonlinear schrödinger equation, Wave motion 29 (4) (1999) 341-361.

[48] P. Kelley, Self-focusing of optical beams, Physical Review Letters 15 (26) (1965) 1005.

[49] V. Talanov, Self-modeling wave beams in a nonlinear dielectric, Radiophysics and Quantum Electronics 9 (2) (1966) 260-261. 
[50] F. Takens, Lecture notes in mathematics, by DA Rand and L.-S. Young Springer, Berlin 898 (1981) 366.

[51] G. Adomian, Nonlinear stochastis system theory and applications to physics kluwer academic publishers (1989). 
Figures
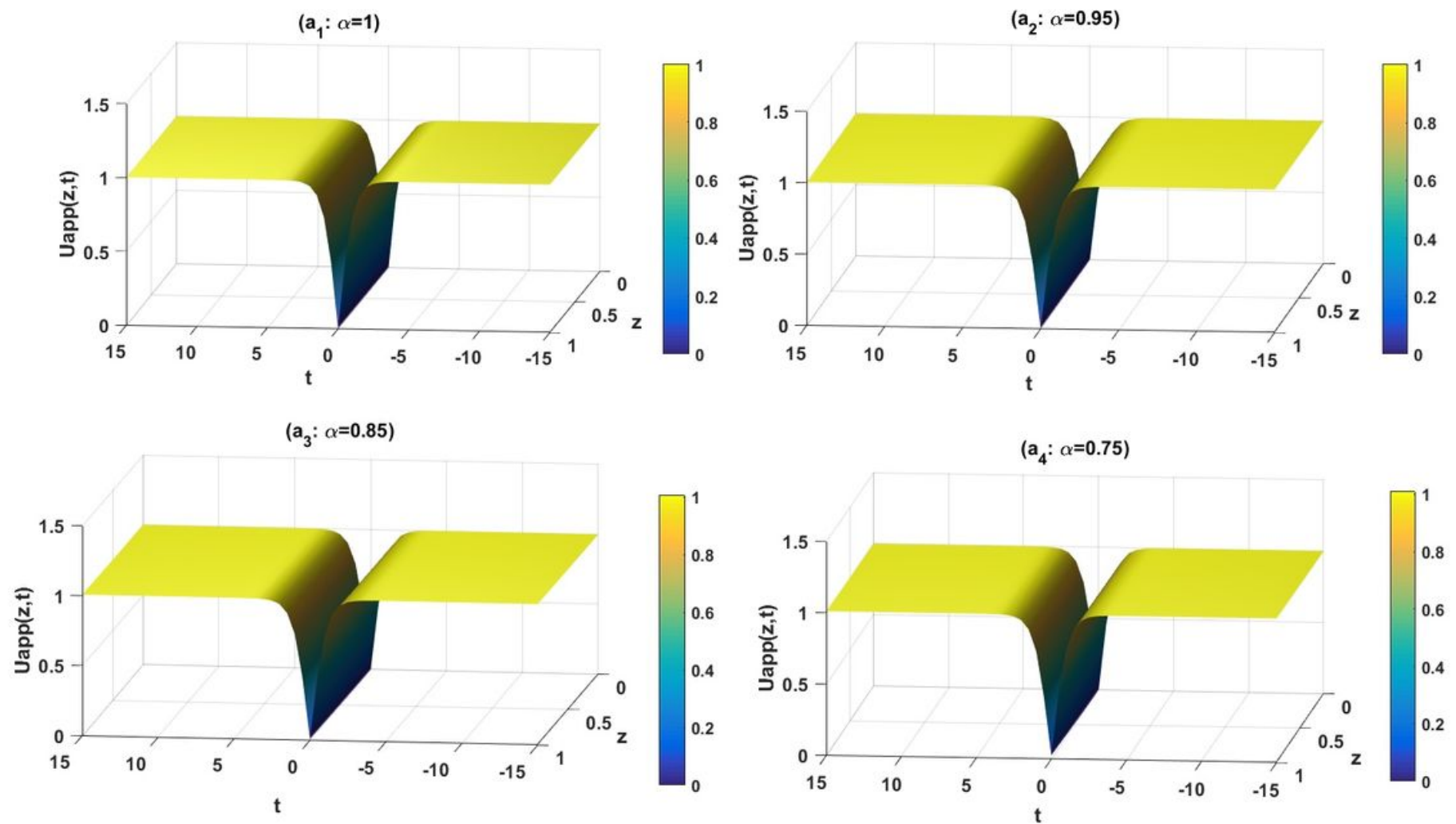

\section{Figure 1}

The approximate dark soliton solution obtained by Adomian decomposition method of the equation (25) (see Manuscript file for full figure legend) 

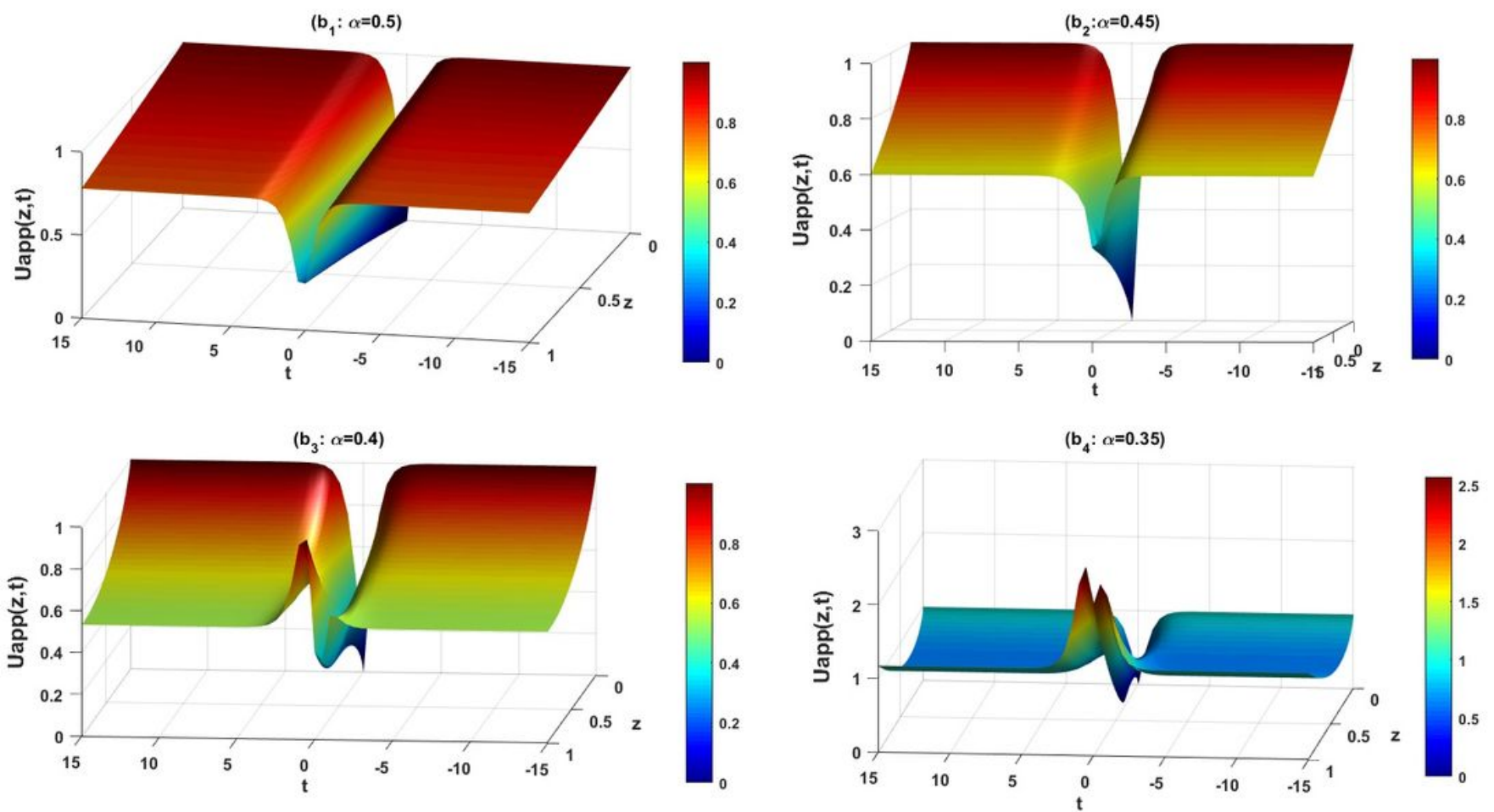

Figure 2

The approximate dark soliton solution obtained by Adomian decomposition method of the equation (25) (see Manuscript file for full figure legend)
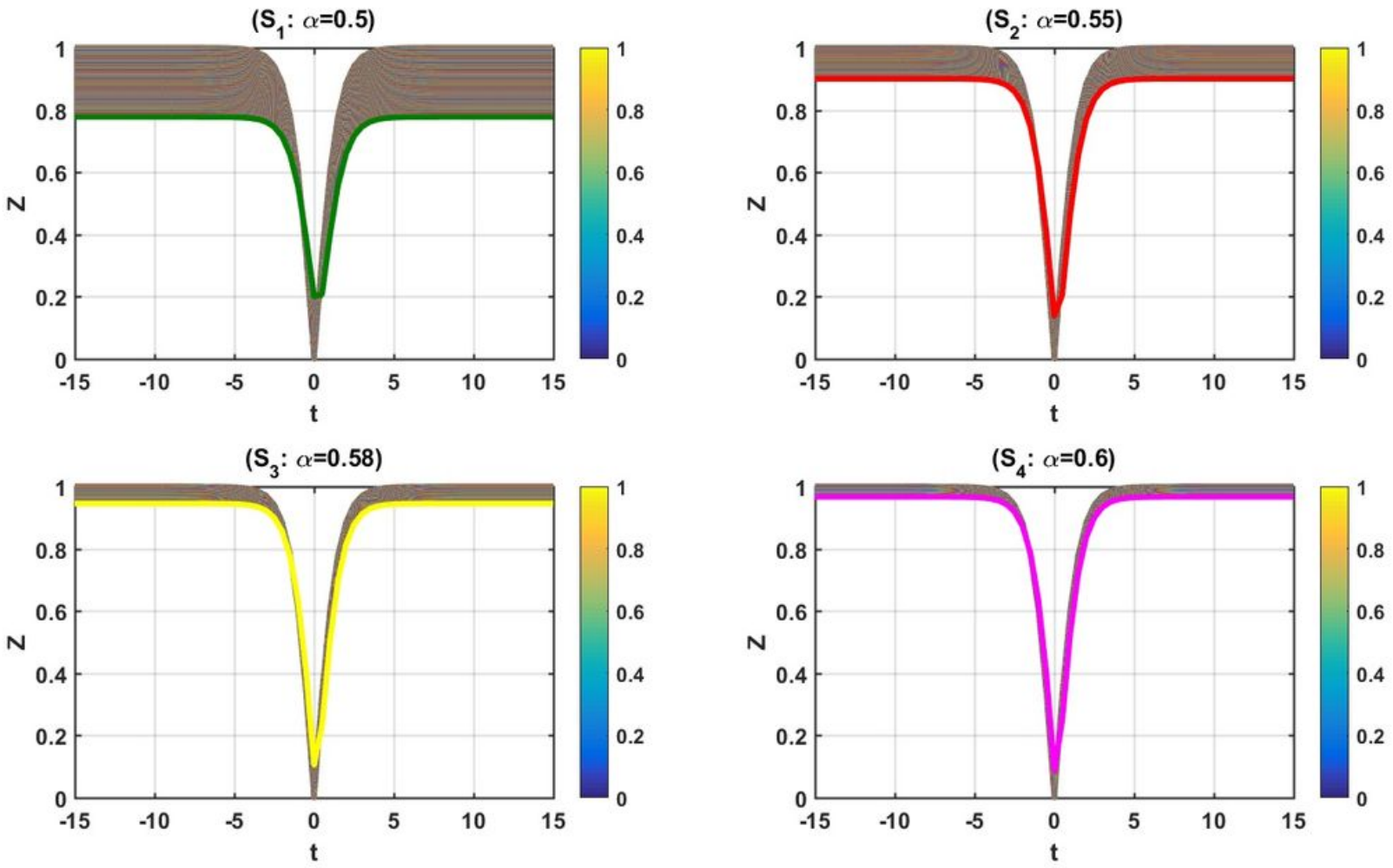

Figure 3 
The approximate dark soliton solution obtained by Adomian decomposition method of the equation (25) (see Manuscript file for full figure legend)

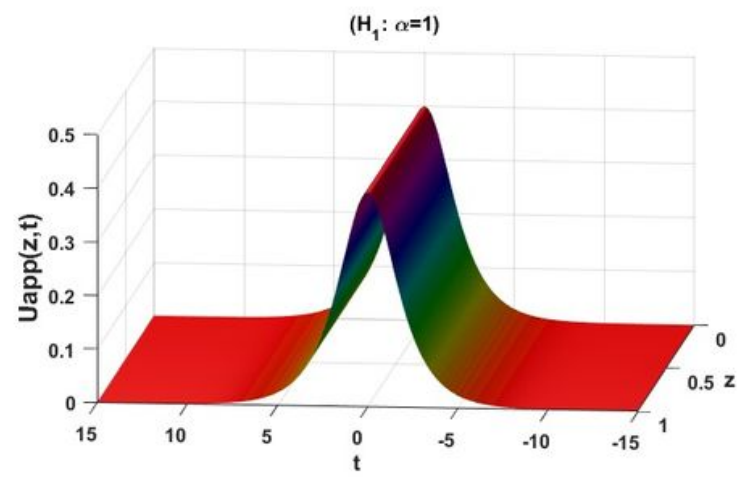

$\left(\mathrm{H}_{3}: \alpha=0.8\right)$

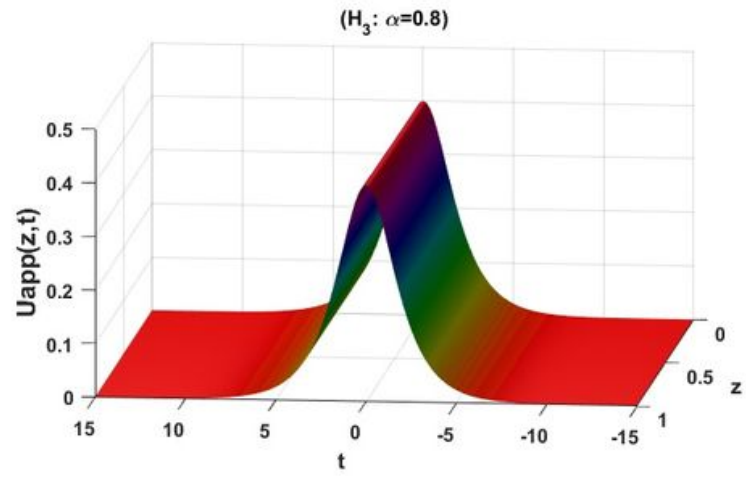

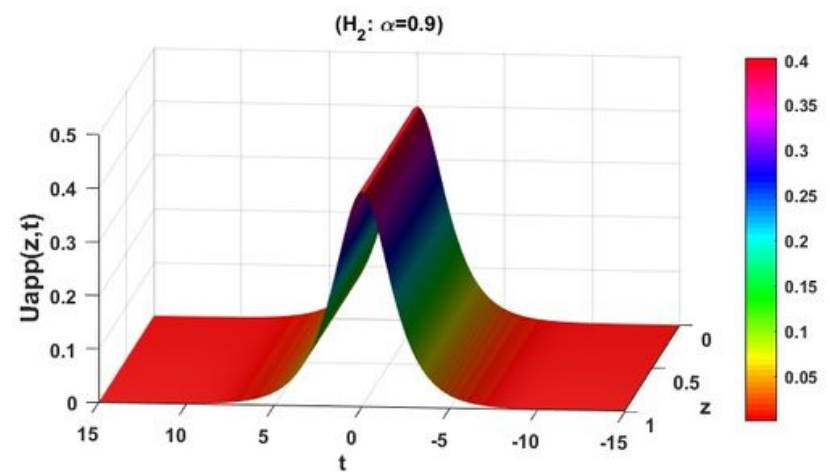

$\left(\mathrm{H}_{4}: \alpha=0.7\right)$

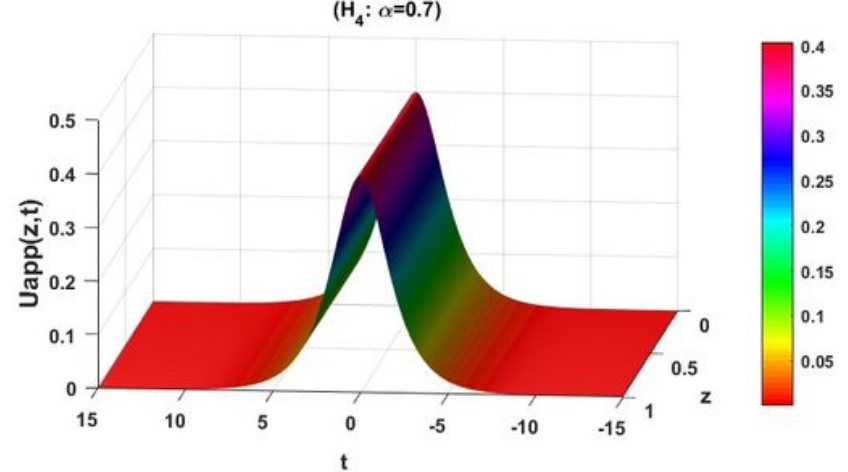

\section{Figure 4}

The approximate bright soliton solution obtained by Adomian decomposition method of the equation (28) (see Manuscript file for full figure legend) 

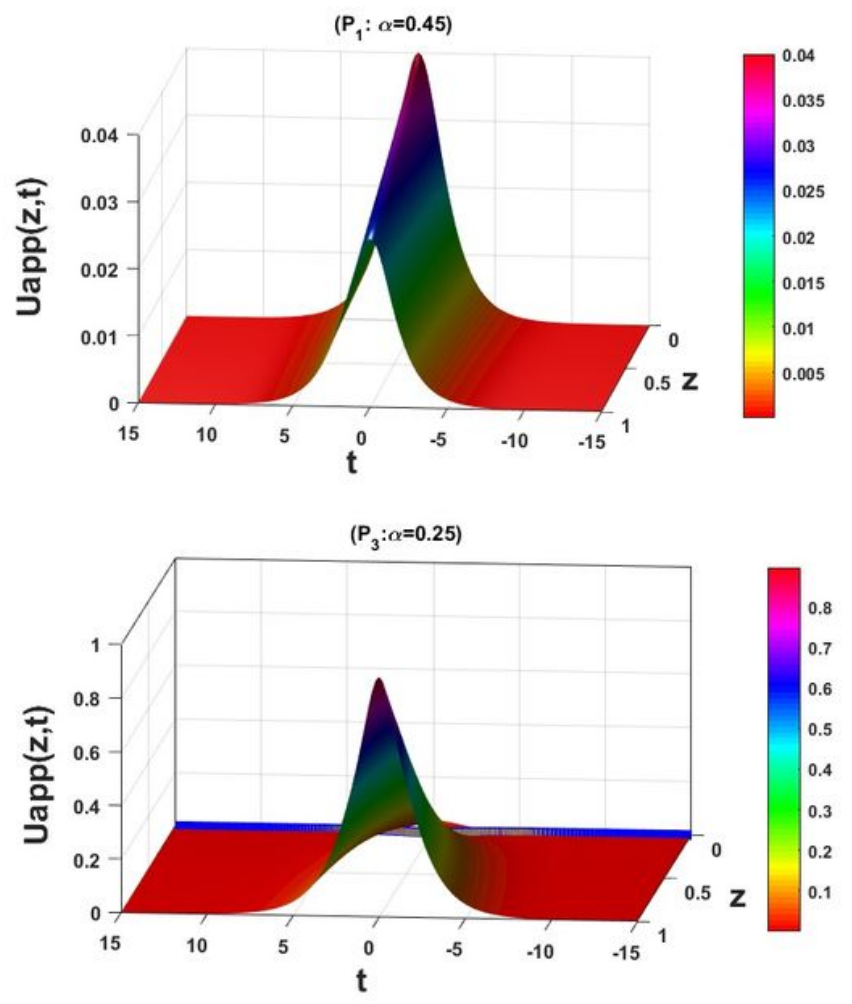

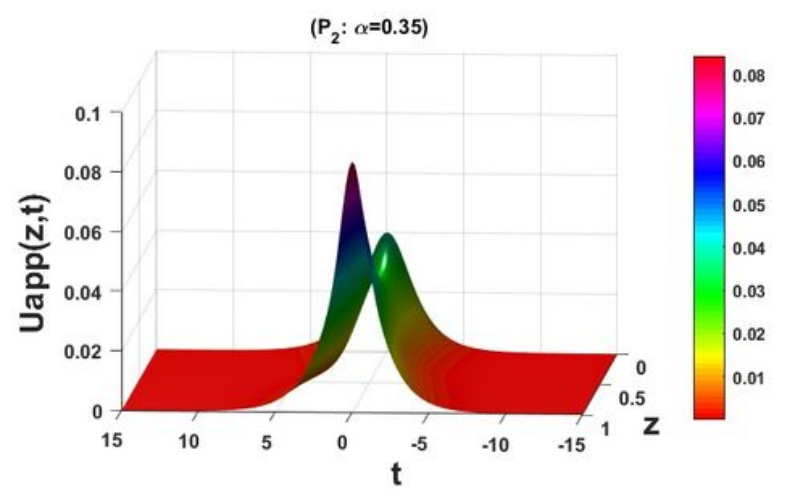

$\left(\mathbf{P}_{4}: \alpha=0.15\right)$

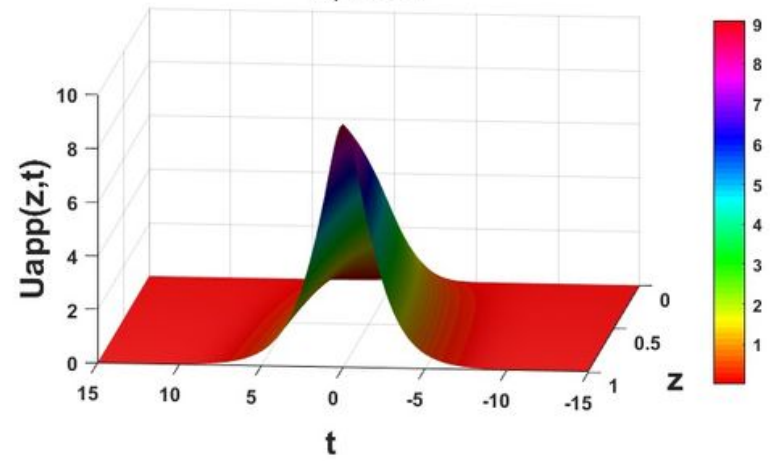

Figure 5

The approximate bright soliton solution obtained by Adomian decomposition method of the equation (28) (see Manuscript file for full figure legend)
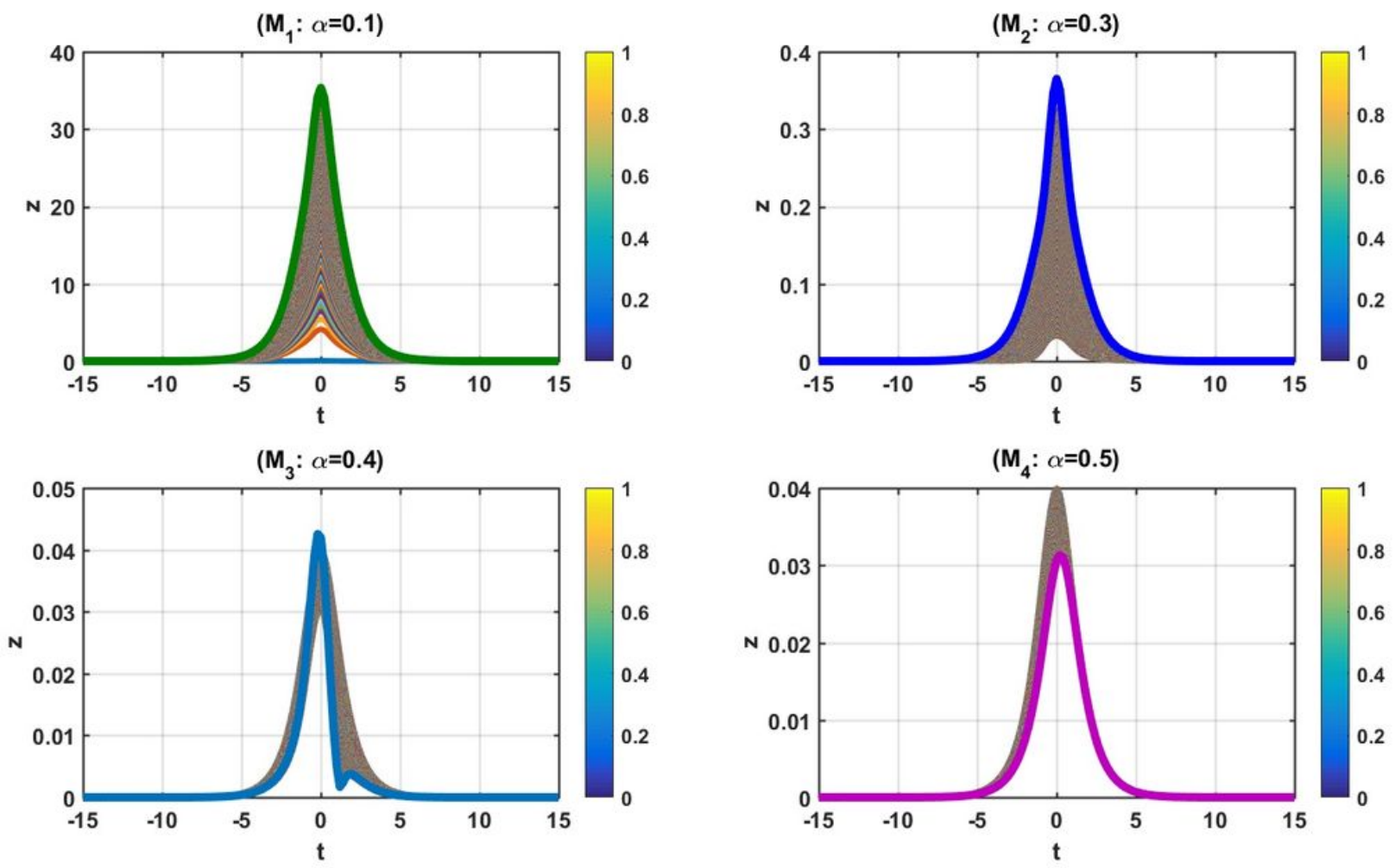
Figure 6

The approximate bright soliton solution obtained by Adomian decomposition method of the equation (28)(see Manuscript file for full figure legend)
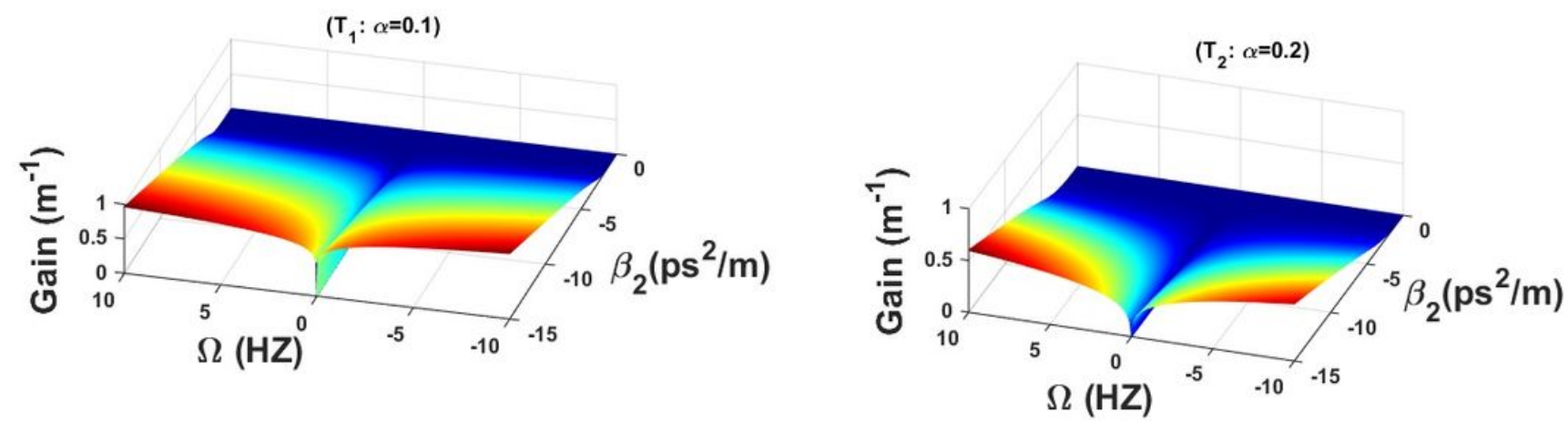

Figure 7

The variation of the (MI) Gain spectrum versus Group velocity dispersion of the (LHNLTL) of equation (35) (see Manuscript file for full figure legend)
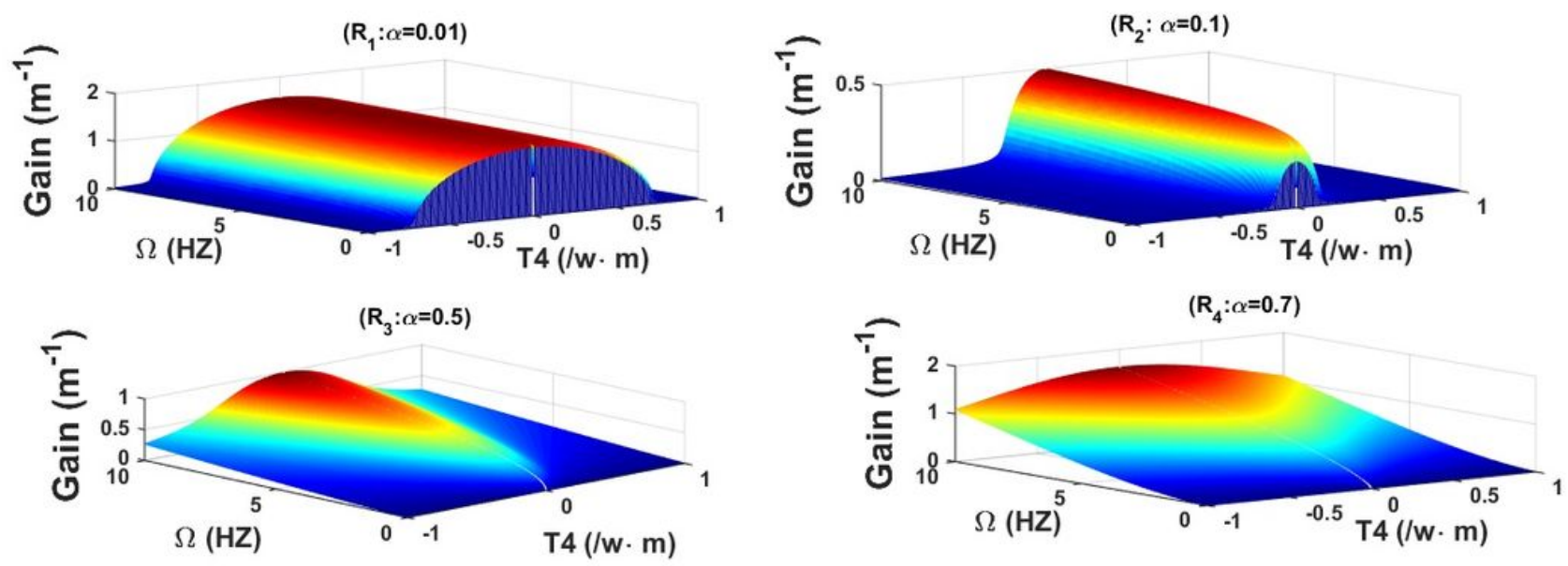

\section{Figure 8}

The variation of the (MI) Gain spectrum versus the kerr nonlinearty of the (LHNLTL) of equation (35) (see Manuscript file for full figure legend) 